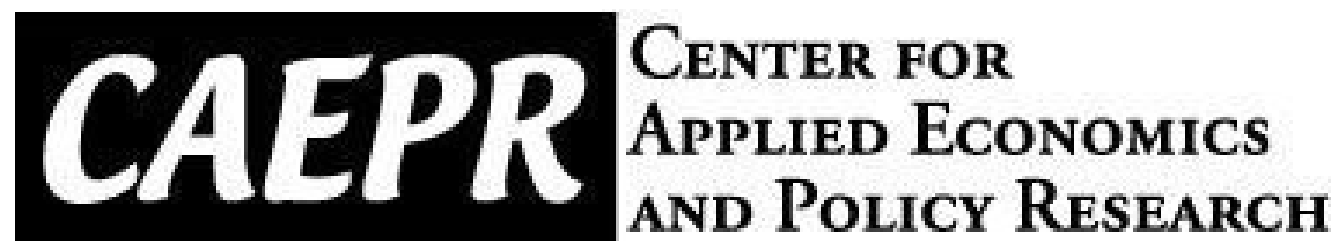

\author{
CAEPR \\ Working Paper \\ \#2017-017
}

\title{
Measuring Asset Market Linkages: Nonlinear Dependence and Tail Risk
}

\author{
Juan Carlos \\ Escanciano \\ Indiana University \\ Javier Hualde \\ Universidad Pública \\ de Navarra
}

December 13, 2017

This paper can be downloaded without charge from the Social Science Research Network electronic library at https://papers.ssrn.com/abstract_id=3088182

The Center for Applied Economics and Policy Research resides in the Department of Economics at Indiana University Bloomington. CAEPR can be found on the Internet at: http://www.indiana.edu/ -caepr. CAEPR can be reached via email at caepr@indiana.edu or via phone at 812-855-4050.

(C)2017 by Juan Carlos Escanciano and Javier Hualde. All rights reserved. Short sections of text, not to exceed two paragraphs, may be quoted without explicit permission provided that full credit, including (C) notice, is given to the source. 


\title{
Measuring Asset Market Linkages: Nonlinear Dependence and Tail Risk*
}

\author{
JuAn Carlos Escanciano ${ }^{\dagger}$ \\ Indiana University
}

\author{
JAVIER HUALDE \\ Universidad Pública de Navarra
}

December 13, 2017

\begin{abstract}
Traditional measures of dependence in time series are typically based on correlations or periodograms. These are adequate in many circumstances but, in others, especially when trying to assess market linkages (e.g., financial contagion), might be inappropriate. In the present paper we propose new nonparametric cumulative measures of dependence and show how they characterize nonparametric persistence, with particular emphasis on measuring tail risk. We propose simple estimates for these measures and establish their limiting properties. We employ the proposed methods to analyze the persistence properties of some of the major international stock market indices during and after the 2007-2009 financial crisis. Our results uncover a leading role of US and London in international diversification. Tail dependence, as quantified with the new measures, is more informative and more robust than the popular Marginal Expected Shortfall for the US, and more persistent than mean dependence. We find an ubiquitous nonlinear persistence in conditional variance across all markets that is not explained by popular parametric models. Market crashes also show substantial persistence.
\end{abstract}

Keywords and Phrases: Nonlinear dependence; Tail risk; Expected Shortfall; Persistence in variance; Market crashes.

JEL Classification: C12, C14.

${ }^{*}$ We thank seminar participants for useful comments at Universidad Carlos III de Madrid, University of Montreal, CESG 2017, the 2017 Summer International Econometrics Workshop at SWUFE, and the 1127 AMS Meeting. We thank Marine Carrasco, Sílvia Gonçalves, Bruce Hansen and Alex Maynard for helpful conversations.

${ }^{\dagger}$ Research funded by the Spanish Plan Nacional de I+D+I, reference number ECO2014-55858-P.

${ }^{\ddagger}$ Research funded by the Spanish Plan Nacional de I+D+I, reference number ECO2015-64330-P. 


\section{Introduction}

Asset market linkages, in particular those related to extreme market events, have been widely studied. These analyses mainly focus on assessing the hypothesis that market correlation is higher in crisis periods, phenomenon also known as financial contagion. For this purpose several testing and estimation strategies have been introduced, including the modelization of the multivariate distribution of extreme returns (Longin and Solnik, 2001), the analysis of the coincidence of extreme returns across different countries (Bae, Karolyi and Stulz, 2003), the estimation of the expected number of market crashes given that at least one market crashes (Hartmann, Straetmans and de Vries, 2004) or the introduction of tools like the Marginal Expected Shortfall (MES) as a measure of systemic risk (Acharya et al., 2017).

Undoubtedly, these proposals, among others, have led to significant improvements in the difficult exercise of measuring tail dependence. Thus, within this setting, the main purpose of the present paper is to define very general measures of dependence and persistence which can easily accommodate previous approaches to assess tail dependence while at the same time offering a richer characterization. Specifically, unlike the previously mentioned approaches, our measures will not just focus on the occurrence of single extreme market events, which are only informative about whether the market is below a certain threshold. In fact, measures of tail dependence based on single extreme market events lead to a relatively narrow characterization of tail risk because they are not informative about how far is the market below the threshold (i.e. they do not account for tail risk). As will be seen, our proposed measures can address this limitation by accounting for a continuum of extreme market events, thereby providing a more complete and robust picture of tail dependence and systemic risk. In particular, this materializes in a new measure of tail dependence, the Maximal Marginal Expected Shortfall (MMES), which is the maximum MES over a set of quantiles in the left tail, and, as such, it is a measure of systemic risk that accounts for tail risk.

More generally, our proposal can be viewed as an attempt to provide useful analytical tools capable of assessing appropriately the dependence and persistence behavior of nonlinear time series (cf. Terasvirta, Tjøstheim, and Granger, 2011). In fact, while in the linear set-up there exists a complete machinery of tools for studying the dynamic properties of such series, mainly based on autocorrelations and periodograms, there are still today relatively few studies on how to measure the persistence properties of nonlinear time series. In fact, this problem is even more accentuated when the interest focuses on inherently nonlinear aspects such as tail dependence. The distinctive 
features of our proposed measures are their simplicity of estimation, implementation and interpretation; their robustness (they are model-free or nonparametric); and their flexibility in adapting to different aspects of the distribution of the underlying time series, in particular tail dependence.

Our programme relates to that of Gallant, Rossi and Tauchen (1993) and Koop, Pesaran and Potter (1996), who extended the impulse-response methodology, initiated by Sims (1980), to a nonlinear framework, see also Gourieroux and Jasiak (1999, 2000), Clarida and Taylor (2003) and Kapetanios (2007) for related approaches. These extensions, however, require either a fully parametric model or nonparametric smoothed estimators of the conditional mean for the time series of interest. The resulting measures of persistence are not operative when the conditioning set considered is high-dimensional or sample sizes are small (such as when estimation is in the tails). In addition, they might depend on user-chosen parameters, such as bandwidths, which substantially affect inference in finite samples. In general, as noted in Gourieroux and Jasiak (1999), the implementation of these approaches requires a fairly large number of observations to give reasonable results, which could rule out the application of these methods to e.g. risk management, for which such large samples are sometimes unavailable.

From a purely theoretical perspective, our very general measures are rooted on the classical literature of nonparametric significance testing in Bierens (1982), Stute (1997), Hong (1999), Escanciano (2006), Escanciano and Velasco (2006a,b), and others. From this viewpoint the main novelty of the paper is to show that certain measures of nonparametric persistence defined in the foundational work of Granger (1995) can be characterized with cumulative dependence measures called Integrated Regression Functions (IRF). Since these measures are extremely simple to estimate, this opens up the possibility of simple measurement of dependence and persistence in a nonparametric setting. Interestingly, restricting the set of integration in the IRF allows focus on certain parts of the distribution. In particular, when integration is restricted to the left tail, the IRF provides a new measure of tail dependence and systemic risk. This flexibility of the IRF was unnoticed in the nonparametric testing literature mentioned above, and is a methodological contribution of this paper. Additionally, we provide novel asymptotic theory for our nonparametric measures under weak dependence conditions, which required extending previous results by Hansen (1996).

As anticipated before, our measures are flexible enough to characterize dependence and persistence linked to any distributional feature, including those related to 
crashes or financial contagion. In particular, we apply these tools to study the persistent effects of the 2007-2009 financial crisis on the major international stock market indices. We are particularly interested in studying the magnitude and persistence of tail events during and after the Great Recession. Our results uncover a leading role of US and London's stock markets for international diversification. Markets in US and London have a short term predictive power for all stocks but for London. The German DAX does not affect the US, has a small impact on the London market, and a significant impact in Asian markets. The latter do not have direct linkages to other markets. We also observe that tail dependence in the US, as quantified with the new measures, is more significant than MES measures, and it is more persistent than mean dependence. We find an ubiquitous nonlinear persistence in conditional variance across all markets, particularly Asian markets. This persistence cannot be explained by popular parametric models such as GARCH models with Student-t distributions (see Francq and Zakoian 2010 for a review of GARCH models). We also look at the persistence of market crashes. We uncover substantial persistence in both the probability of a crash and the number of crashes. The magnitude and persistence of systemic risk is very sensitive to the severity of losses of individual markets, i.e. to individual tail risk. Overall, our results contribute to a better understanding of international asset market linkages and international diversification, and provide a more robust measurement of dependence and persistence than traditional measures based on linear correlations or single extreme market events.

The layout of the article is as follows. In Section 2 we introduce our dependence measures, illustrate their usefulness to capture many different forms of dependence and persistence, with special emphasis on those related to extreme events, making also connections with existing measures of systemic risk. Section 3 provides theoretical support to our measures, relating them to previous attempts to measure nonlinear dependence by Granger (1995, 2003). A natural extension to capture distributional dependence is introduce and we also discuss several estimation issues. Section 4 reports the results of the empirical application, and in Section 5 we conclude and describe further research. A mathematical Appendix establishes asymptotic theory for the estimators and gathers all proofs.

\section{Nonlinear Measures of Dependence}

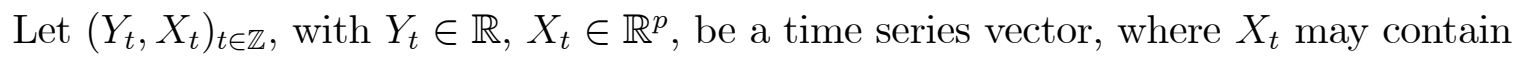
lagged values of $Y_{t}$. Also, let $E\left(Y_{t}\right)=\mu_{t}$ for all $t$. Extensions to multivariate $Y_{t}$ 
are straightforward, and hence they are not considered for the sake of exposition. A natural way of characterizing persistence and dependence is to define the $h$-step regression function $m_{t, h}(x)=E\left(Y_{t+h} \mid X_{t}=x\right), x \in \mathbb{R}^{p}, h \geq 0$, almost surely (a.s.), which can be estimated nonparametrically. For example, Robinson (1983) has studied the large sample properties of kernel estimators of $m_{t, h}$ for various lags $h$, see also Auestad and Tjøstheim (1990). Inference on these functions, however, requires smoothing estimation involving bandwidth choices. Moreover, smoothed estimators of $m_{t, h}$ are very sensitive to the dimension $p$, a feature that is referred to as the curse of dimensionality.

The proposed measures of dependence avoid smoothing by considering integrals of the regression functions $m_{t, h}$. Define the IRF as

$$
\gamma_{t, h}(x):=\operatorname{Cov}\left(Y_{t+h}, 1\left(X_{t} \leq x\right)\right)=E\left[\left(Y_{t+h}-\mu_{t+h}\right) 1\left(X_{t} \leq x\right)\right]
$$

where $1(A)$ denotes the indicator function of the event $A$. These measures were introduced in Escanciano and Velasco (2006a) for time series; see also Stute (1997) for the different purpose of nonparametric significance testing in regression with independent data. Note that

$$
\gamma_{t, h}(x)=\int_{-\infty}^{x}\left(m_{t, h}(z)-\mu_{t+h}\right) F_{t}(d z),
$$

where $F_{t}$ is the cumulative distribution function (cdf) of $X_{t}$, which justifies the name for $\gamma_{t, h}$. A "large" value of $\gamma_{t, h}$ suggests a large difference between the conditional mean $m_{t, h}$ and the unconditional one $\mu_{t+h}$, which in turn indicates that $Y_{t+h}$ and $X_{t}$ are dependent in mean. In order to measure the "size" of $\gamma_{t, h}$ we introduce the uniform (sup-) norm $\left\|\gamma_{t, h}\right\|_{\infty}=\sup _{x \in \mathbb{R}^{p}}\left|\gamma_{t, h}(x)\right|$. We illustrate the behavior of our measures by means of several examples.

Example 1 (Mean Dependence). Let $Z_{t}$ be a sequence of strictly stationary, mean-zero, Gaussian random variables (r.v.'s) with $r(h)=E\left[Z_{t} Z_{t+h}\right], h \geq 1, r(0)=1$. It can be easily shown that, choosing $Y_{t}=X_{t}=Z_{t}$,

$$
\gamma_{t, h}(x)=r(h) \int_{-\infty}^{x} z(2 \pi)^{-1 / 2} \exp \left(-0.5 z^{2}\right) d z, \quad h \geq 1, x \in \mathbb{R}^{p} .
$$

Therefore we have $\left\|\gamma_{t, h}\right\|_{\infty} \sim C r(h)$, for a constant $C$. Thus, under Gaussianity and mild regularity conditions, our measure of dependence reduces, as expected, to the traditional linear one.

In other situations the object of interest is not the mean of the process but some 
higher order moments, e.g. volatility. The next example illustrates how our measures can be easily adapted to cover this case.

Example 2 (Mean and Volatility Dependence). Let

$$
Z_{t}=c+\phi Z_{t-1}+\varepsilon_{t}
$$

where $-1<\phi<1$, and $\varepsilon_{t}$ is conditionally (on the past) Gaussian with zero mean and variance $\sigma_{t}^{2}$, where

$$
\sigma_{t}^{2}=a+\alpha \varepsilon_{t-1}^{2}
$$

with $a>0,0<\alpha<1$, so $Z_{t}$ follows an $\mathrm{AR}(1)$ with $\mathrm{ARCH}(1)$ errors model. Gallant et al. (1993) analyzed the persistence properties of this model by means of its conditional mean and volatility profiles for the case $c=0$. Setting $Y_{t}=Z_{t}, X_{t}=\left(Z_{t}, Z_{t-1}\right)^{\prime}$, the persistence in conditional mean depends on

$$
\gamma_{t, h}(x)=\int_{-\infty}^{\infty} \int_{-\infty}^{\infty}\left[E\left(Y_{t+h}-\mu_{t+h} \mid X_{t}=z\right)\right] 1(z \leq x) F(d z)
$$

where $F$ is the cdf of $X_{t}$. It can be easily shown that

$$
\gamma_{t, h}(x)=\phi^{h} \operatorname{Cov}\left(Y_{t}, 1\left(X_{t} \leq x\right)\right)
$$

so if $0<E\left[\left|Y_{t}\right|\right]<\infty,\left\|\gamma_{t, h}\right\|_{\infty}$ converges to zero at the exact rate $\phi^{h}$. Additionally, the persistence in second moments can be captured by setting $Y_{t}=Z_{t}^{2}, X_{t}=\left(Z_{t}, Z_{t-1}\right)^{\prime}$. For these choices it can be shown that

$$
\begin{aligned}
E\left(Y_{t+h} \mid X_{t}=z\right)= & \frac{a}{1-\alpha} \sum_{j=1}^{h}\left(1-\alpha^{j}\right) \phi^{2(h-j)}+\frac{c^{2}\left(1-\phi^{h}\right)^{2}}{(1-\phi)^{2}}+\frac{2 c \phi^{h}\left(1-\phi^{h}\right)}{1-\phi} z_{1} \\
& +\left(z_{1}-c-\phi z_{2}\right)^{2} \sum_{j=1}^{h} \alpha^{j} \phi^{2(h-j)}+\phi^{2 h} z_{1}^{2}
\end{aligned}
$$

where $z=\left(z_{1}, z_{2}\right)^{\prime}$. Noting

$$
E\left(Y_{t+h}\right)=\frac{a}{(1-\alpha)\left(1-\phi^{2}\right)}+\frac{c^{2}}{(1-\phi)^{2}}
$$


then

$\gamma_{t, h}(x)=\int_{-\infty}^{\infty} \int_{-\infty}^{\infty}\left(-\rho_{1}(h)-\rho_{2}(h)+\rho_{3}(h) z_{1}+\rho_{4}(h)\left(z_{1}-c-\phi z_{2}\right)^{2}+\phi^{2 h} z_{1}^{2}\right) 1(z \leq x) F(d z)$,

where

$$
\begin{aligned}
\rho_{1}(h) & \sim K_{1}(a, \alpha, \phi)\left(\phi^{2 h} 1\left(\phi^{2}>\alpha\right)+h \phi^{2 h} 1\left(\phi^{2}=\alpha\right)+\alpha^{h} 1\left(\phi^{2}<\alpha\right)\right), \\
\rho_{i}(h) & \sim K_{i}(c, \phi) \phi^{h}, i=2,3 \\
\rho_{4}(h) & \sim K_{4}(\alpha, \phi)\left(\phi^{2 h} 1\left(\phi^{2}>\alpha\right)+h \phi^{2 h} 1\left(\phi^{2}=\alpha\right)+\alpha^{h} 1\left(\phi^{2}<\alpha\right)\right)
\end{aligned}
$$

and $K_{i}$ in (2) are constants depending on the specified parameters. Assuming $0<$ $E\left[Y_{t}\right], E\left[\varepsilon_{t}^{2}\right]<\infty$, the results in $(2)$ determine the exact rates at which $\left\|\gamma_{t, h}\right\|_{\infty}$ converges to zero as $h$ increases, which basically depend on the relation between the ARCH parameter $\alpha$ and the AR parameter $\phi$. If $\phi<\alpha,\left\|\gamma_{t, h}\right\|_{\infty}$ decreases at the exact rate $\alpha^{h}$; if $\phi^{2}<\alpha<\phi$, this rate is $\phi^{h}$, but only due to the presence of $c \neq 0$ in (1), which induces persistence in the squares, the rate being $\alpha^{h}$ if $c=0$; if $\alpha<\phi^{2}$ (assuming $c=0$ ), the rate is $\phi^{2 h}$; finally, if $\alpha=\phi^{2}$ (with $c=0$ ), $Z_{t}$ is more persistent in volatility than if $\alpha \neq \phi^{2}$, with a rate of decrease given by $h \alpha^{h}$. Essentially, apart from the effect of the constant, the AR structure of the model generates persistence driven by $\phi^{2}$, whereas that implied by the ARCH structure depends on $\alpha$, so our measure captures which one of these effects dominates. In contrast with the volatility impulse response function of Gallant et al. (1993), which was specifically designed to analyze the second moment properties of the process (separately from first moment properties), our measure of persistence of volatility capture first and second moment effects. In our view, this is not a drawback of our approach, because the parameter $\phi$ has influence in the persistence of the squares (as it is the case when there are not ARCH effects).

The IRFs in the previous examples focus on conditional mean and volatility dependence and persistence. However in many applications the interest centers around other components of the distribution. In fact, as the next example shows, it is possible to connect our measures with well known measures of systemic risk and contagion, and in doing so, we extend existing measures to more informative measures that better account for tail risk. Let $x_{\text {tail }}$ denote some threshold that defines the region of 
interest. Then, the restricted sup (pseudo)-norm

$$
\left\|\gamma_{t, h}\right\|_{\infty, x_{t a i l}}=\sup _{x \in\left(-\infty, x_{\text {tail }}\right]}\left|\gamma_{t, h}(x)\right|
$$

can be applied to $\gamma_{t, h}(x)$ to define tail dependence (precisely, how $X_{t}$ being in the left tail affects the mean of $\left.Y_{t+h}\right)$. Interestingly, the tail-restricted IRF is closely related to a recent and popular measure of systemic risk, the MES, since

$$
\gamma_{t, h}(x)=\left(M E S_{t, h}(x)-\mu_{t+h}\right) F_{t}(x)
$$

where $M E S_{t, h}(x)=E\left(Y_{t+h} \mid X_{t} \leq x\right)$. The MES of Acharya et al. (2017) corresponds to $M E S_{t, 0}(x)$ with $x$ equals the $\alpha$-quantile of the distribution $F_{t}$, for the market return index $X_{t}$. By focusing on a continuum of values of $x$, rather than just one, our measure $\left\|\gamma_{t, h}\right\|_{\infty, x_{\text {tail }}}$ better captures tail dependence, and as such, contains more information on tail risk than existing measures.

The next example shows how we can adapt our methodology to examine the predictability and persistent properties of market crashes. The phenomenon of how market crashes spill over to other countries was first systematically studied by Morgenstern (1959) and it has been extensively studied after that. The new measures provide useful tools to study crashes and their dynamic and systemic properties. We will particularize them to two stocks (whose returns are denoted as $r_{t, 1}, r_{t, 2}$ ), although generalizations are straightforward.

Example 3 (Persistence of Crashes). Choose $r_{\text {crash }}^{(1)}$ so that we define a market crash at time $t$ whenever $r_{t, 1} \leq r_{\text {crash }}^{(1)}\left(r_{\text {crash }}^{(1)}\right.$ could be, e.g., the $1 \%$ or $5 \%$ empirical quantile). Set $Y_{t}=1\left(r_{t, 1} \leq r_{c r a s h}^{(1)}\right), X_{t}=r_{t, 2}$. Then, noting (3),

$$
E\left(Y_{t+h} \mid X_{t} \leq x\right)=\operatorname{Pr}\left(r_{t+h, 1} \leq r_{c r a s h}^{(1)} \mid r_{t, 2} \leq x\right)=F_{t+h}^{(1)}\left(r_{c r a s h}^{(1)}\right)+\frac{\gamma_{t, h}(x)}{F_{t}^{(2)}(x)}
$$

where $F_{t}^{(1)}, F_{t}^{(2)}$, are the cdf's of $r_{t, 1}, r_{t, 2}$, respectively. Thus the conditional probability of a crash equals the unconditional one plus a correction term which captures the tail dependence between $r_{t+h, 1}$ and $r_{t, 2}$. This is mainly characterized by our measure, which for this particular case equals

$$
\gamma_{t, h}(x)=\operatorname{Pr}\left(r_{t+h, 1} \leq r_{c r a s h}^{(1)}, r_{t, 2} \leq x\right)-\operatorname{Pr}\left(r_{t+h, 1} \leq r_{c r a s h}^{(1)}\right) \operatorname{Pr}\left(r_{t, 2} \leq x\right)
$$

A simple modification of $Y_{t}$ leads to a measure of dependence for the number of crashes. Choose $r_{c r a s h}^{(2)}$ so that we define a market crash at time $t$ whenever $r_{t, 2} \leq r_{\text {crash }}^{(2)}$. 
Define $Y_{t}=1\left(r_{t, 1} \leq r_{\text {crash }}^{(1)}\right)+1\left(r_{t, 2} \leq r_{\text {crash }}^{(2)}\right)$, so $Y_{t}$ corresponds to the number of crashes at time $t$. Then $E\left(Y_{t+h} \mid X_{t} \leq x\right)$ relates closely to the extreme market linkage indicator of Hartmann, Straetmans and de Vries (2004). Nicely, setting again $X_{t}=r_{t, 2}$

$$
E\left(Y_{t+h} \mid X_{t} \leq x\right)=F_{t+h}^{(1)}\left(r_{\text {crash }}^{(1)}\right)+F_{t+h}^{(2)}\left(r_{\text {crash }}^{(2)}\right)+\frac{\gamma_{t, h}(x)}{F_{t}^{(2)}(x)}
$$

where, again, our measure accounts for tail dependence.

Finally, set $Y_{t}=1\left(r_{t, 1} \leq r_{\text {crash }}^{(1)}\right) 1\left(r_{t, 2} \leq r_{\text {crash }}^{(2)}\right)$, so $E\left(Y_{t+h} \mid X_{t} \leq x\right)$ represents the probability of simultaneous crashes given a tail event for $r_{t, 2}$. Denoting by $F_{t}^{(1,2)}$ the joint cumulative distribution function of $r_{t, 1}, r_{t, 2}$, and setting $X_{t}=r_{t, 2}$, it can be easily shown that

$$
E\left(Y_{t+h} \mid X_{t} \leq x\right)=F_{t+h}^{(1,2)}\left(r_{c r a s h}^{(1)}, r_{c r a s h}^{(2)}\right)+\frac{\gamma_{t, h}(x)}{F_{t}^{(2)}(x)}
$$

Then $\|\cdot\|_{\infty, x_{t a i l}}$ applied to (4), (5), (6) would define appropriate measures of crash persistence and dependence.

\section{Theoretical Framework and Estimation}

\subsection{Characterization of Persistence}

In this section we present some of the theoretical properties of our measures, we additionally introduce a natural generalization and finally we discuss some estimation issues. First we explore the connection between our measures a very general notion of nonparametric persistence in mean proposed by Granger (1995). Following Granger (1995), we define the concepts of Short Memory in Mean (SMM) and Extended Memory in Mean (EMM) as follows.

Definition 1. $\left(Y_{t}, X_{t}\right)_{t \in \mathbb{Z}}$ is called $S M M$ if for all $t \in \mathbb{Z}$

$$
\lim _{h \rightarrow \infty} E\left(m_{t, h}\left(X_{t}\right)-\mu_{t+h}\right)^{2}=0 .
$$

If $\left(Y_{t}, X_{t}\right)_{t \in \mathbb{Z}}$ does not satisfy the previous condition is called EMM.

Granger (1995) allowed for $p=\infty$, but to make this definition practically operative $p<\infty$ is needed, although potentially $p$ can be large. Granger and Hallman (1991) replaced the name of EMM by long memory in mean, which could be slightly 
misleading because, in general, a stationary linear long memory process is SMM. Gourieroux and Jasiak (1999) referred to SMM and EMM as nonlinear integrated and nonlinear integrated of order zero, respectively. As noted by Granger (1995) the concepts of SMM and EMM are related to a kind of "mixing in mean" property, or, more precisely, to the concept of mixingale (cf. McLeish, 1974); see Davidson (1994, Chapter 16) and the Appendix here.

We shall show that, under a mild moment condition,

$$
\lim _{h \rightarrow \infty} E\left(m_{t, h}\left(X_{t}\right)-\mu_{t+h}\right)^{2}=0 \Longleftrightarrow \lim _{h \rightarrow \infty}\left\|\gamma_{t, h}\right\|_{\infty}=0
$$

This new characterization justifies measuring nonparametric persistence with the measures $\gamma_{t, h}$.

Theorem 1. If $\sup _{t} E Y_{t}^{2}<\infty$, then $\left(Y_{t}, X_{t}\right)_{t \in \mathbb{Z}}$ is SMM iff $\lim _{h \rightarrow \infty}\left\|\gamma_{t, h}\right\|_{\infty}=0$.

\subsection{Extension to Distributional Dependence}

In some instances, the interest is in the whole joint distribution of $\left(Y_{t+h}, X_{t}\right)$. Granger (2003) defines a persistent process in distribution using the bivariate and marginal densities at different lags. Gourieroux and Jasiak (2002) considered series expansions estimators for the nonlinear canonical analysis of the series, cf. Buja (1990). In this paper we propose alternative measures that avoid ill-posed problems and bandwidth choices.

Let $f_{t, h}(y, x), k_{t+h}(y)$, and $g_{t}(x)$ be, respectively, the joint and marginal densities of $Y_{t+h}$ and $X_{t}$. Following Granger (2003), we define

$$
s_{t, h}(y, x)=f_{t, h}^{1 / 2}(y, x)-k_{t+h}^{1 / 2}(y) g_{t}^{1 / 2}(x) \quad h \geq 1
$$

Definition 2. $\left(Y_{t}, X_{t}\right)_{t \in \mathbb{Z}}$ is called short memory in distribution (SMD) if for all $t \in \mathbb{Z}$

$$
\lim _{h \rightarrow \infty} \iint_{\mathbb{R} \times \mathbb{R}^{d}}\left|s_{t, h}(y, x)\right|^{2} d y d x=0 .
$$

If $\left(Y_{t}, X_{t}\right)_{t \in \mathbb{Z}}$ does not satisfy previous condition is called extended memory in distribution (EMD).

Alternative definitions can be given in terms of other divergence measures or distances, see Granger, Maasoumi and Racine (2004) and references therein. The function $s_{t, h}$ and related quantities could be estimated by plugging in nonparametric 
smoothed estimators of the densities, see McDonald, Shalizi and Schervish (2015) for a related approach. Such estimators, however, require bandwidth choices and are highly sensitive to the dimension $p$.

Following the logic above, we propose using the cumulative measure

$$
\begin{aligned}
\eta_{t, h}(y, x) & =\operatorname{Cov}\left(1\left(Y_{t+h} \leq y\right), 1\left(X_{t} \leq x\right)\right) \\
& =F_{t, h}(y, x)-K_{t+h}(y) G_{t}(x)
\end{aligned}
$$

where $F_{t, h}(y, x), K_{t+h}(y)$, and $G_{t}(x)$ are, respectively, the bivariate and marginal cdf's of $Y_{t+h}$ and $X_{t}$, so for the choice $X_{t}=Y_{t}, \eta_{t, h}(y, x)$ corresponds to the Laplace cross-covariance kernel of Dette et al. (2015). Then, we have the following extension of Theorem 1 . With some abuse of notation, let $\|\cdot\|_{\infty}$ denote the supremum norm extended to functions of $(y, x)$.

Theorem 2. $\left(Y_{t}, X_{t}\right)_{t \in \mathbb{Z}}$ is $S M D$ iff $\lim _{h \rightarrow \infty}\left\|\eta_{t, h}\right\|_{\infty}=0$.

We illustrate the behavior of these measures by means of two examples.

Example 4. Taking $Z_{t}$ as in Example 1 and setting $Y_{t}=X_{t}=Z_{t}$, from Appendix 2 in Gourieroux and Jasiak (2002),

$$
\eta_{t, h}(y, x)=r(h) E\left[Z_{t} 1\left(Z_{t} \leq x\right)\right] E\left[Z_{t} 1\left(Z_{t} \leq y\right)\right]+o(r(h)), \quad h \geq 1, x \in \mathbb{R} .
$$

Thus we have $\left\|\eta_{t, h}\right\|_{\infty} \sim C r(h)$, for a constant $C$.

Again, under Gaussianity and additional regularity conditions, our concept of persistence in distribution captures the same dependence as linear measures. More generally, by Hoeffding's identity (see Hoeffding, 1940), when $p=1$

$$
\operatorname{Cov}\left(Y_{t+h}, X_{t}\right)=\int \eta_{t, h}(y, x) d y d x
$$

from which is clear that $\eta_{t, h}$ also contains the information from the linear dependence in the data.

Again, we could tailor $\eta_{t, h}(y, x)$ to specific values of $y$ and $x$ to focus on different parts of the distribution. For example, when $y$ and $x$ are set in the left tail of the distributions of $Y_{t+h}$ and $X_{t}$, respectively, the covariances $\eta_{t, h}(y, x)$ measure distributional tail dependence. Specifically, we define the tail dependence measure

$$
\left\|\eta_{t, h}\right\|_{\infty, y_{\text {tail }}, x_{\text {tail }}}=\sup _{y \leq y_{\text {tail }}} \sup _{x \leq x_{\text {tail }}}\left|\eta_{t, h}(y, x)\right| .
$$


This extends the linear correlation measures defined in Longin and Solnik (2001), Ang and Bekaert (2002), Ang and Chen (2002), and Bae, Karolyi and Stulz (2003), which have been used to define contagion in financial markets, and which correspond to setting $y$ and $x$ to a single value equal to an empirical quantile, such as the $5 \%$. Considering a continuum of values of $y$ and $x$ bring much more information on distributional tail dependence. Our measure of tail dependence is different from tail dependence measures defined in the literature on copulas. To see this, define $\alpha_{y}=K_{t+h}\left(y_{t a i l}\right)$ and $\alpha_{x}=G_{t}\left(x_{t a i l}\right)$, and let $C_{t, h}$ denote the copula function of $Y_{t+h}$ and $X_{t}($ with $p=1)$, i.e. $C_{t, h}(u, v)=F_{t, h}\left(K_{t+h}^{-1}(u), G_{t}^{-1}(v)\right)$ when $K_{t+h}$ and $G_{t}$ are strictly monotone (see Malevergne and Sornette 2006 for an extensive discussion of copulas and tail dependence measures). Then, our measure can be written as

$$
\left\|\eta_{t, h}\right\|_{\infty, y_{t a i l}, x_{t a i l}}=\sup _{0 \leq u \leq \alpha_{y}} \sup _{0 \leq v \leq \alpha_{x}}\left|C_{t, h}(u, v)-u v\right| .
$$

This is different from the commonly used tail dependence measure

$$
\lambda_{t, h}:=\lim _{u \rightarrow 0} \frac{C_{t, h}(u, u)}{u}
$$

which only contains information on what happens in the limit, where few data is available. Nonparametric estimation of $\lambda_{t, h}$ is challenging, and requires smoothing of the data in the tails. In contrast, as shown below, nonparametric estimation of $\left\|\eta_{t, h}\right\|_{\infty, y_{t a i l}, x_{t a i l}}$ is straightforward and it does not require bandwidth choices.

\subsection{Estimation}

We discus now how the new measures are estimated. For simplicity in the exposition, we consider strictly stationary time series, although our results allow for extensions to non-stationary sequences, applying the results of e.g. Andrews and Pollard (1994) and Hansen (1996). The natural estimator for $\gamma_{h} \equiv \gamma_{t, h}$ based on a sample $\left\{Y_{t}, X_{t}\right\}_{t=1}^{n}$ is

$$
\widehat{\gamma}_{h}(x)=\frac{1}{n-h} \sum_{t=1}^{n-h}\left(Y_{t+h}-\bar{Y}_{n-h}\right) 1\left(X_{t} \leq x\right),
$$

with $\bar{Y}_{n-h}=(n-h)^{-1} \sum_{t=1}^{n-h} Y_{t}$. Replacing $Y_{t}$ by $\sigma_{Y}^{-1} Y_{t}$, where $\sigma_{Y}^{2}=E\left(Y_{t}-\mu\right)^{2}$ and $\mu=E\left(Y_{t}\right)$, will make the measure $\gamma_{t, h}$ scale invariant. To improve interpretation 
sometimes we consider a correlation-type process

$$
\widehat{\rho}_{h}(x)=\frac{\widehat{\gamma}_{h}(x)}{\sqrt{\widehat{\sigma}_{n, h}^{2} \hat{F}_{h}(x)\left(1-\hat{F}_{h}(x)\right)}},
$$

where

$$
\widehat{\sigma}_{n, h}^{2}=\frac{1}{n-h} \sum_{t=1}^{n-h}\left(Y_{t+h}-\bar{Y}_{n-h}\right)^{2} \text { and } \hat{F}_{h}(x)=\frac{1}{n-h} \sum_{t=1}^{n-h} 1\left(X_{t} \leq x\right)
$$

Correlation-type processes may have denominators that are close to zero, so some care must be exercised when defining dependence based on them.

To measure persistence we estimate functionals such as $\left\|\gamma_{t, h}\right\|_{\infty}$ or its scaled version with Kolmogorov-Smirnov (KS) type tests $K S_{n}(h)$ given by

$$
K S_{n}(h):=\sup _{x \in \mathcal{R}}\left|\widehat{\rho}_{h}(x)\right| \quad h \geq 1
$$

where $\mathcal{R}=\left\{x \in \mathbb{R}: \hat{F}_{h}^{-1}(0.01) \leq x \leq \hat{F}_{h}^{-1}(0.99)\right\}$ trims the data to avoid dividing by zero in $\widehat{\rho}_{h}$. We note that trimming is not necessary if $\widehat{\rho}_{h}$ is replaced by $\widehat{\gamma}_{h}$. Since both $\widehat{\gamma}_{h}(x)$ and $\hat{F}_{h}(x)$ are step functions the computation of $K S_{n}(h)$ simplifies to computing the maximum over a finite set of points $\left(X_{t}\right.$ in $\left.\mathcal{R}\right)$. Code to compute our measures is available from the authors upon request.

Tail dependence measures for systemic risk can be estimated analogously. Standardizing $Y_{t+h}$, i.e. $Y_{t+h}^{s}=\sigma_{Y}^{-1}\left(Y_{t}-\mu\right), M_{E} S_{t, h}(x)=E\left(Y_{t+h}^{s} \mid X_{t} \leq x\right)$ is estimated by

$$
\widehat{M E S}_{h}(x)=\frac{\widehat{\gamma}_{h}(x)}{\widehat{\sigma}_{n, h} \hat{F}_{h}(x)},
$$

and the MMES is computed as

$$
S R_{n}(h):=\sup _{x \in \mathcal{T}}\left|\widehat{M E S}_{h}(x)\right| \quad h \geq 1
$$

where $\mathcal{T}$ is a set in the left tail distribution, e.g. $\mathcal{T}=\left\{x \in \mathbb{R}: \hat{F}_{h}^{-1}(0.01) \leq x \leq\right.$ $\left.\hat{F}_{h}^{-1}(0.05)\right\}$. Again, computation of $S R_{n}(h)$ is simplified to a maximum over a finite set of points (data points $X_{t}$ in $\mathcal{T}$ ), and if $\hat{F}_{h}(x)$ is dropped from the denominator the supremum can be extended to the whole left tail (e.g. $-\infty \leq x \leq \hat{F}_{h}^{-1}(0.05)$ ). 
Distributional measures of dependence are similarly estimated as

$$
\widehat{\eta}_{h}(y, x)=\frac{1}{n-h} \sum_{t=1}^{n-h} 1_{c}\left(Y_{t+h}, y\right) 1_{c}\left(X_{t}, x\right)
$$

where $1_{c}\left(Z_{t}, x\right)=1\left(Z_{t} \leq x\right)-(n-h)^{-1} \sum_{s=1}^{n-h} 1\left(Z_{s} \leq x\right)$, for a generic series $\left\{Z_{t}\right\}$.

The asymptotic properties of the estimated measures $\widehat{\gamma}_{h}$ and $\widehat{\eta}_{h}$, and other related quantities, are investigated in the Appendix. There, sufficient conditions are given for weak convergence of these estimates as stochastic processes. See van der Vaart and Wellner (1996) for a comprehensive review of the corresponding theory for independent observations. The limiting distributions under weak dependence assumptions depend on the data generating process in a complicated way. This means that asymptotic critical values for quantities such as (9) are not readily available. To overcome this problem we use resampling methods. A simple bootstrap approximation of the measures $\gamma_{h}(x)$ is given by

$$
\widehat{\gamma}_{h}^{*}(x)=\frac{1}{n-h} \sum_{t=1}^{n-h}\left(Y_{t+h}-\bar{Y}_{n-h}\right) 1_{c}\left(X_{t}, x\right) V_{t}
$$

where $V_{t}$ is a sequence of independent r.v.'s with zero mean, unit variance, bounded support and also independent of the sequence $\left\{Y_{t}, X_{t}\right\}_{t=1}^{n}$. This procedure is similar to the wild bootstrap used in $\mathrm{Wu}(1986)$. Examples of $\left\{V_{t}\right\}$ sequences are iid Bernoulli variates with $P\left(V_{t}=0.5(1-\sqrt{5})\right)=(1+\sqrt{5}) / 2 \sqrt{5}$ and $P\left(V_{t}=0.5(1+\sqrt{5})\right)=1-$ $(1+\sqrt{5}) / 2 \sqrt{5}$. With the bootstrap critical values we can compute uniform confidence bands for $\widehat{\gamma}_{h}$. Another resampling method that works under general conditions is subsampling, see the monograph by Politis, Romano and Wolf (1999).

\section{Asset Market Linkages and Tail Risk During and After the Financial Crisis.}

In this section we apply our methodology to study the nonlinear dependence and persistence properties of some of the major international stock market indices during and after the recent financial crisis. We aim to investigate three different features of these data sets. First, we study the nonlinear dependence in mean properties. This also includes persistence of tail events on the mean using the new measures of tail dependence. Second, we compare nonparametric measures of persistence in conditional variance with parametric ones based on GARCH. Finally, we employ a 
particular version of our results to analyze the persistence of market crashes during and after the financial crisis.

The data consist in daily closed stock returns for S\&P500 (SP500), Dow Jones Industrials (DJ), Frankfurt DAX Index (DAX), the London FTSE-100 Index (FTSE), Tokyo Nikkei Index (NIK) and the Hong Kong Hang Seng Index (HANG). The daily data are taken from January 4, 2007 to June 30, 2016. The daily closed values for these stock indices are obtained from Yahoo finance. ${ }^{1}$ We consider the returns of the indices obtained as the log differences of the data, which exclude dividend payments. Figure 1 plots the data and Table 1 provides basic descriptive statistics.

$$
\text { FIGURE } 1 \text { AND TABLE } 1 \text { ABOUT HERE }
$$

All stock returns have small means with large kurtosis, particularly in the US. This is explained by the large extreme events observed during the financial crisis. Stock returns are typically linearly unpredictable at a daily frequency, but the reported results for linear correlations reveal that US stock returns present significant negative correlations at the first lag. We also report p-values for testing serial correlation based on the data-driven version of the Box-Pierce test in Escanciano and Lobato (2009). The null of lack of correlation is clearly rejected for US stocks at $1 \%$ and at $5 \%$ for Nikkei, but fails to be rejected for other stocks. Unreported results show little or no persistence in return's correlations, as is commonly observed. Thus, classical linear dependence measures do not provide much information on the dependence structure in the data. Additionally, it is known that linear correlations are biased upwards in periods of high volatility (cf. Forbes and Rigobon, 2002), which make them inappropriate to study market dependencies in periods such as the recent financial crisis.

\subsection{Market Linkages and persistence in conditional mean}

The martingale properties of individual stocks returns have been extensively investigated in the literature, see e.g. Lo and MacKinlay (1999) and references therein. These earlier studies showed that stock price changes are not martingale difference sequences (MDS), although recent evidence supporting or refuting the martingale hypothesis for some stock indices seems mixed; see e.g. Escanciano and Velasco (2006b) with the S\&P500. Our results below provide extensive evidence on the predictability of stock returns during this period, albeit with little or no persistence in conditional

\footnotetext{
${ }^{1}$ FTSE was not available through Yahoo and it was obtained from Bloomberg.
} 
mean.

To gain insight on the mean dependence properties of the stock returns, and then on the asset market linkages during the financial crisis and its aftermath, we report in Table 2 the bootstrap p-values computed with the wild-bootstrap approximation described in (12) for the KS test in (9), for testing the significance of the measure $\gamma_{h}$ at the first lag $h=1$ and all combinations of stocks. ${ }^{2}$ We observe that US and FTSE have a significant predictive power in predicting mean returns for all stocks but for the FTSE. The inability of other stocks to affect London's market can be useful for an international investor seeking to diversify risk. The German DAX does not affect the US, has a small impact on the London's market, and a significant impact on Asian markets, which in turn do not have significant predictive power on any market. Of course, the high predictability at one lag of Asian markets from other markets, in particular US markets, can be explained by the different time zones in which they function. These results draw a mapping of nonlinear directional predictability that might be useful for international diversification and hedging; see Christoffersen et al. (2012) for the importance of accounting for nonlinear dependence in international diversification.

Of particular concern to international investors is international systemic risk in times when a market crashes. Table 3 reports the bootstrap p-values for the MMES tail risk measures at the first lag when the conditioning stock market is in its left tail, accounting for all quantiles between the $1 \%$ and $5 \%$. The conclusions are qualitatively similar to those of Table 2. The robustness of the London's stocks to the extreme events of the US market is of significant interest. For completeness, we report in Table 4 the same bootstrap p-values when only the $5 \%$ quantile is considered, that is, using a dynamic version of the MSE measure of Acharya et al. (2017). It is interesting to see how focusing on just one quantile misses much of the information that is used to predict US stocks. This illustrates the benefits of the new measures of tail risk that, by accounting for a continuum of quantiles, use more information on the tails.

The results of Tables 2-4 contribute to the literature of prediction of stock returns, providing substantial evidence of nonlinear predictability during the financial crisis and its aftermath, and suggesting a leading role of the London's stock market for international diversification in the short run. Whether these conclusions hold for longer term investments is something we turn in next.

\section{TABLES 2-4 ABOUT HERE}

\footnotetext{
${ }^{2}$ Whenever we compare two stocks we restrict samples to days where both stocks are open, to avoid biases coming from non-synchronism.
} 
Although there is substantial evidence of predictability at one lag, there is overall little or no persistence in the conditional mean of the data. We provide empirical evidence of the little predictability in mean by plotting in Figure 2 the Nonlinear AutoCorrelogram (NAC), that is the function $K S_{n}(h)$ as a function of $h$, when we condition on the SP500 for lags up to 300. This plot can be useful for detecting nonlinearities graphically and can play the same role as the usual autocorrelograms in the linear setup. Figure 2 suggests little persistence in mean. Figures 3 and 4 report the NAC for tail risk, as measured by the MMES, when we condition on tail events for the SP500 and FTSE, respectively. Tail dependence is more persistent than mean dependence. For example, tail events of the SP500 have significant effects on the German DAX even after 120 business days. These effects, although large in magnitude, are cyclical and not systematic. Interestingly, FTSE seems to have larger short term effects on other markets than the SP500, although they seem to be less persistent. Overall, we find little persistence in mean and little persistence in tail dependence, from the tail of one stock market to the mean return level of another stock market, and particularly from US, where the crisis originated, to other countries.

FIGURES 2-4 ABOUT HERE

\subsection{Persistence in conditional variances}

By modern asset pricing theory the conditional variance of future asset returns is a key component of the price of an asset. Thus, analyzing the persistence properties of conditional variances is of primary importance for a risk manager in order to take asset allocation decisions. In particular, the extent to which shocks to conditional variances have short or long-run effects is crucial to determine the risk premium of long-term contracts. These ideas have been the subject of an extensive theoretical and empirical literature; see e.g. Poterba and Summers (1986), who gave evidence of the linkage between persistence of conditional variances and stock prices, or the seminal contribution of King and Wadhwani (1989) who proposed a structural model of volatility transmission and investigated its empirical content for the October 1987 crash.

Much of the existing literature has been restricted to linear persistence, see, for instance, the AR(1) model used in Poterba and Summers (1986) or the integrated 
GARCH (IGARCH) model suggested by Engle and Bollerslev (1986), see also Lin, Engle and Ito (1994). The use of parametric models might hide important nonlinear sources of persistence. Thus, in this subsection we study the nonparametric persistent properties in conditional variances of the stocks considered, and we also measure the extent to which linear and nonlinear persistent measures differ. To that end, we apply our new methodology and consider the estimates in (9) where $Y_{t+h}$ is the squared return and $X_{t}$ is the return.

According to our previous results, most of the conditional means are not significantly different from zero. Therefore, the measures $\widehat{\gamma}_{h}$ can indeed be interpreted as measures of nonlinear persistence in the conditional variance. In cases where the conditional mean is not zero, Example 2 suggests that $\widehat{\gamma}_{h}$ still measures persistence in variance (as persistence in variance dominates persistence in mean). There are already two well-known stylized facts that have been extensively documented in the literature of financial time series; first, conditional variances are time-varying; and second, stock returns and other financial time series are quite persistent in conditional second moments (cf. Engle and Bollerslev, 1986, Bollerslev and Engle, 1993), especially when large sample sizes are considered. Linear measures of dependence were sufficient to uncover these two important stylized facts, but, alternatively, we ask the following questions: is nonlinear persistence different from linear persistence? If so, to what extent are they different?

We made an attempt to clarify these issues by means of the following experiment, which basically analyzes whether linear components of the conditional variance persistence (captured by means of parametric models) account for all persistence (in volatility) present in the data. Thus, initially an $\operatorname{AR}(1)-\operatorname{GARCH}(1,1)$ model with Student-t innovations was fitted to each data set, and we used the estimated parameters to simulate data of the same sample size as the original data. Then, we estimate our nonparametric measures of dependence with the simulated data. In this way, we can asses if parametric models are able to explain the nonlinear persistence present in the data. To save space, we only present the results for the SP500, but all other stocks lead qualitatively to the same conclusions. We report in Figure 5 the nonparametric KS tests statistics at different lags and the wild bootstrap 95\% quantile for $K S_{n}(h):=\sup _{x \in \mathcal{R}}\left|\widehat{\rho}_{h}(x)\right|$, jointly with KS tests from the parametric fits and the $95 \%$ confidence interval.

\section{FIGURE 5 ABOUT HERE}

From our results we strongly reject that parametric models such as the AR-GARCH 
model with Student-t distribution are able to replicate the results of our nonparametric measures of persistence in volatility. We find that nonlinear persistence is considerably higher than linear persistence for all stocks (results for other stocks are available upon request). In unreported experiments we observed that this conclusion is robust to different specifications of the volatility model, such as an EGARCH and AGARCH specifications (see Francq and Zakoian 2010 for details on these specifications).

Table 5 reports the number of rejections of tests of constant volatility for all stocks, and confirms the leading role of the Chinese HANG in risk spillovers, particularly on US stocks. Interestingly, cross-border linkages are not weaker than domestic linkages. The inability of parametric models to explain nonparametric volatility persistence is robust to the exclusion of the 2007-2009 crisis period (results on subsamples can be obtained from the authors upon request).

\section{TABLE 5 ABOUT HERE}

\subsection{Persistence of crashes}

We define a market crash as the indicator of the market being below its $5 \%$ empirical quantile, and take this as dependent variable

$$
Y_{t+h, i}=1\left(r_{t+h, i} \leq \hat{F}_{h, i}^{-1}(0.05)\right)
$$

where $r_{t+h, i}$ is the return of the stock $i-t h$ at time $t+h$ and $\hat{F}_{h, i}^{-1}(0.05)$ the empirical $5 \%$ quantile of the sample $\left\{r_{t, i}\right\}_{t=1+h}^{n}$. This implies that estimates of

$$
M E S_{h, i j}(x)=E\left(Y_{t+h, i} \mid r_{t, j} \leq x\right)
$$

are estimates of the probability of a crash in market $i, h$ periods ahead, when market $j$ is below the threshold $x$. One possibility is to use $x=\hat{F}_{h, j}^{-1}(0.05)$, which relates to the co-exceedances used in Bae, Karolyi and Stulz (2003) and provides a further application of the MES measures proposed in Acharya et al. (2017). As mentioned above, a more informative measure about tail risk uses a continuum of $x$ in the left tail. For example, we consider the maximal conditional probability estimates

$$
C_{n, i j}(h):=\sup _{x \in \mathcal{T}} \widehat{M E S}_{h, i j}(x) \quad h \geq 1
$$


where $\mathcal{T}=\left\{x \in \mathbb{R}: \hat{F}_{h, j}^{-1}(0.01) \leq x \leq \hat{F}_{h, j}^{-1}(0.05)\right\}$ and

$$
\widehat{M E S}_{h, i j}(x)=\frac{\sum_{t=1}^{n-h} 1\left(r_{t+h, i} \leq \hat{F}_{h, i}^{-1}(0.05)\right) 1\left(r_{t, j} \leq x\right)}{\sum_{t=1}^{n-h} 1\left(r_{t, j} \leq x\right)}
$$

Figures 6-8 plot $C_{n, i j}(h)$ for some representative values of $i$ and $j$ ( $j=$ SP500, DAX and HANG). We observe estimated conditional probabilities of crashes well above the unconditional probability of 5\% (in many cases above 30\%). Furthermore, market crashes are quite persistent.

\section{FIGURES 6-8 ABOUT HERE}

We also report results for the number of crashes. Defining $Y_{t+h}$ equals the number of crashes at time $t+h$, i.e.

$$
Y_{t+h}=1\left(r_{t+h, 1} \leq \hat{F}_{h, 1}^{-1}(0.05)\right)+\cdots+1\left(r_{t+h, 6} \leq \hat{F}_{h, 6}^{-1}(0.05)\right),
$$

one can measure the predictability and persistent properties of the total number of crashes. Hartmann, Straetmans and de Vries (2004) studied nonparametric estimation of the contemporaneous expected number of market crashes conditional on at least one market crashing. We look at dynamics and condition on particular stocks to facilitate interpretation. That is, we measure dynamic systemic risk; how a crash in a particular market at time $t$ propagates in the whole system $h$ periods ahead and leads to a systemic crash, as measured by the total number of crashes. Figure 9 plots the maximum expected value of number of crashes conditional on crashes for different stocks, i.e. the estimates

$$
N C_{n, j}(h):=\sup _{x \in \mathcal{T}} \widehat{M E S}_{h, j}(x) \quad h \geq 1
$$

where $\mathcal{T}=\left\{x \in \mathbb{R}: \hat{F}_{h, j}^{-1}(0.01) \leq x \leq \hat{F}_{h, j}^{-1}(0.05)\right\}, Y_{t+h}$ is defined in (13) and

$$
\widehat{M E S}_{h, j}(x)=\frac{\sum_{t=1}^{n-h} Y_{t+h} 1\left(r_{t+h, j} \leq x\right)}{\sum_{t=1}^{n-h} 1\left(r_{t+h, j} \leq x\right)}
$$

\section{FIGURE 9 ABOUT HERE}

Interestingly, the estimated number of crashes shows an inverted-u pattern, where first increases with the number lags, reaching a maximum after 30-40 business days, 
for latter decreasing monotonically towards its unconditional value of 0.3 . US stocks, SP500 and DJ, and the HANG lead to the more persistent effects in crashes, with a number of rejections at lags 200-300 of 14, 12 and 11, respectively. Figure 9 also

plots the expected number of crashes conditional on a marginal crash at $5 \%$ (a MES measure). The MMES is significantly much larger than the MES for all stocks at all lags, and more persistent. More extreme tail events than those observed at the $5 \%$ level have substantially larger and more persistent effects. This point nicely illustrates the practical benefits of accounting for a continuum of risk levels in the proposed measures, rather than just focusing on a single one, as is common in the risk management literature, and which misses much information on the tail risk involved.

\section{Conclusions and Summary}

In this paper we have introduced new simple measures of nonlinear dependence. In contrast to related approaches, our measures are easy to estimate, do not need of large sample sizes and are nonparametric in nature, without relying on user-chosen parameters (like bandwidths). Thus, we have proposed generally applicable tools for measuring nonlinear dependence in macroeconomic and financial data. The proposed measures are easily adapted to situations where tail dependence is of interest. We have established connections with existing measures of systemic risk and show in an application that the proposed measures provide a more accurate description of the tail risk and its persistent properties during and after the recent financial crisis than their counterparts based on single market events.

Another empirical finding is the ubiquitous nonlinear persistence in conditional variance that is not explained by popular parametric models. Although asset market linkages in mean follow a specific directional pattern, from US and London to other markets, volatility linkages are bidirectional, with cross-border linkages being as important as domestic linkages. These findings have important economic implications in, e.g., asset pricing and hedging. In particular, our findings show that current shocks to conditional variances may have a permanent effect on long term contracts.

Linear measures can substantially understate the actual (nonlinear) persistence present in economic data. One can argue that some of the existing puzzles in economics and finance might be explained by this notorious limitation of linear methods; see for instance the so-called equity premium puzzle (inability of many asset pricing models in explaining the $6 \%$ annual equity premium and its standard deviation of $19 \%$ ). The growing literature of long-run risks emphasizes the fundamental role of 
persistence of consumption growth on the equity premium to explain these asset pricing anomalies; see Bansal and Yaron (2004). Our new tools can be used to assess to what extent these linear models of asset pricing are able to "fit" the nonlinear persistence of asset prices and consumption growth, as well as the relation between them.

Our work also complements, and has the potential to make operative, some of the ideas presented in Bollerslev and Engle (1993) in nonparametric situations. Our empirical findings suggest that stock market returns can be extended memory in variance, and may present co-persistent properties, as suggested in Bollerslev and Engle (1993). The economic consequences of these important findings will be investigated elsewhere.

Formal inferential procedures for testing the short and extended memory character of a given time series are also of paramount importance, and deserve further investigation. Moreover, our operative measures pave the way for a formal definition of cointegration in nonlinear set-ups that can formalize some of the ideas put forward by previous studies. We leave these and other important extensions of our methods for future research. 


\section{Appendix}

\subsection{Asymptotic theory}

This section provides formal asymptotic theory for the estimators of the new measures. For simplicity in the exposition, we restrict ourselves to strictly stationary and ergodic sequences, although our results allow for extensions to some non-stationary or nonergodic sequences, as in e.g. Andrews and Pollard (1994) and Hansen (1996). Under the strictly stationary assumption, the natural estimators for $\gamma_{h} \equiv \gamma_{t, h}$ and $\eta_{h} \equiv \eta_{t, h}$, based on a sample $\left\{Y_{t}, X_{t}\right\}_{t=1}^{n}$ are given in (8) and (11), respectively. In this section we establish the asymptotic limits of these estimated measures $\widehat{\gamma}_{h}$ and $\widehat{\eta}_{h}$. Since these are random functions, to develop the asymptotic theory we consider results from empirical processes theory as discussed, for instance, in van der Vaart and Wellner (1996). Throughout the rest of the paper the lag $h \geq 1$ is fixed. Define $1_{e}\left(X_{t} \leq x\right) \equiv 1\left(X_{t} \leq x\right)-E\left[1\left(X_{t} \leq x\right)\right]$. We consider $\widehat{\gamma}_{h}$ as a process in $\ell^{\infty}\left(\overline{\mathbb{R}}^{p}\right)$, where $\overline{\mathbb{R}}^{p}:=[-\infty, \infty]^{p}$, by extending its definition as $\widehat{\gamma}_{h}(+\infty)=0=\widehat{\gamma}_{h}(-\infty)$. We denote $\Longrightarrow$, the weak convergence in $\left(\ell^{\infty}(\Pi), d_{\infty}\right)$ in the sense of J. HoffmannJørgensen (see Definition 1.3.3 in van der Vaart and Wellner, 1996). Throughout, $A^{\prime}$ and $|A|$ denote the matrix transpose and the Euclidean norm of $A$, respectively. $\|A\|_{p}$ denotes the usual $L_{p}$ norm for a random variable (r.v.), i.e. $\|A\|_{p}=\left(E|A|^{p}\right)^{1 / p}$, and with an abuse of notation we denote by $L_{p}$ the set of r.v.'s with $\|A\|_{p}<\infty$.

Under strictly stationarity and ergodicity, the uniform consistency of $\widehat{\gamma}_{h}(\cdot)$ or $\widehat{\eta}_{h}(\cdot)$ follows from the Ergodic Theorem and a Glivenko-Cantelli's argument, see e.g. Koul and Stute (1999). Thus, this section focusses on the weak convergence of

$$
v_{n, h}(\cdot)=\sqrt{n-h}\left(\widehat{\gamma}_{h}(\cdot)-\gamma_{h}(\cdot)\right) \quad \text { and } \quad \omega_{n, h}(\cdot)=\sqrt{n-h}\left(\widehat{\eta}_{h}(\cdot)-\eta_{h}(\cdot)\right),
$$

under weak dependence assumptions. We establish the asymptotic theory for $v_{n, h}(\cdot)$ and $\omega_{n, h}(\cdot)$ under two different dependence scenarios, namely, mixingale classes and strong mixing processes. These weak dependence concepts are specially adapted to our SMM and SMD concepts, respectively. We also discuss the special case of no dependence (martingale difference sequences and iid processes) as corollary of our main results. With these asymptotic results, we can compute uniform confidence bands for $\widehat{\gamma}_{h, w}, \widehat{\eta}_{h, w}$, and the significance of $\gamma_{h, w}, \eta_{h, w}$, can be tested.

A well-known result in the econometrics and statistical literature is that the weak convergence of a process $R_{n, h}(\cdot)$ (e.g. $v_{n, h}(\cdot)$ or $\left.\omega_{n, h}(\cdot)\right)$ in $\left(\ell^{\infty}(\Pi), d_{\infty}\right)$ will follow from the following conditions (see e.g. p. 2251 in Andrews, 1994): 
(i) $(\Pi, \rho)$ is a totally bounded pseudometric space, for some pseudometric $\rho$;

(ii) $\left(R_{n, h}\left(z_{1}\right), \ldots, R_{n, h}\left(z_{m}\right)\right)$ converges in distribution to a Borel law as $n \rightarrow 0$, for all fixed points $z_{1}, \ldots, z_{m}$ in $\Pi$;

(iii) $\left\{R_{n, h} ; n \geq 1\right\}$ is stochastically equicontinuous, i.e., for every $\varepsilon, \eta>0$ there exists a $\delta>0$ such that

$$
\underset{n \rightarrow \infty}{\operatorname{Limsup} P^{*}}\left[\sup _{z_{1}, z_{2}: \rho\left(z_{1}, z_{2}\right)<\delta}\left|R_{n, h}\left(z_{1}\right)-R_{n, h}\left(z_{2}\right)\right|>\varepsilon\right]<\eta
$$

Examples of pseudometrics in condition (i) are typically

$$
\rho_{m, h}\left(x_{1}, x_{2}\right)=\left(E\left[\left(Y_{t+h}-\mu\right)^{2}\left|1_{e}\left(X_{t} \leq x_{1}\right)-1_{e}\left(X_{t} \leq x_{2}\right)\right|^{2}\right]\right)^{1 / 2}
$$

and

$$
\rho_{d, h}\left(z_{1}, z_{2}\right)=\left(E\left[\left|1_{e}\left(Y_{t+h} \leq y_{1}\right) 1_{e}\left(X_{t} \leq x_{1}\right)-1_{e}\left(Y_{t+h} \leq y_{2}\right) 1_{e}\left(X_{t} \leq x_{2}\right)\right|^{2}\right]\right)^{1 / 2}
$$

for $v_{n, h}(\cdot)$ and $\omega_{n, h}(\cdot)$, respectively, where $z_{i}=\left(y_{i}, x_{i}\right), i=1,2$.

To prove weak convergence to a Gaussian process we must verify that conditions (i-iii) hold. Condition (i) usually follows under mild continuity assumptions on the marginal and joint distribution functions. Conditions implying (ii) are abundant in the literature; see e.g. de Jong (1997) and Dedecker, Merlevede and Volny (2007) for mixingales and Ango Nze and Doukhan (2004) for strong mixing processes. Usually, the most difficult condition to verify is the stochastic equicontinuity in (iii).

\subsubsection{Asymptotic theory under mixingales}

This subsection introduces the concept of a mixingale class and establish the weak convergence of $v_{n, h}$ for mixingale classes. The use of mixingales is motivated by their close relation to our concept of SMM, as can be seen from the following definition introduced by Hansen (1996). Define $I_{t}=\left(X_{t}^{\prime}, X_{t-1}^{\prime}, \ldots\right)^{\prime}$ and let $\mathcal{F}_{t}$ and $\mathcal{H}_{t}$ be the $\sigma$-fields generated by $I_{t}$ and $H_{t}=\left(Y_{t}, X_{t}^{\prime}, Y_{t-1}, X_{t-1}^{\prime}, \ldots\right)^{\prime}$, respectively.

Definition 3. $\left\{f(\cdot), \mathcal{H}_{t}, \mathcal{Q}\right\}_{t \in \mathbb{Z}}$ is called an $L^{q}$-mixingale class, $q \geq 1$, if there exist sequences of nonnegative constants $\left\{c_{t}(f)\right\}_{t \in \mathbb{Z}}$ and $\left\{\zeta_{m}\right\}_{m \in \mathbb{N}}$ such that $\zeta_{m} \rightarrow 0$ as $m \rightarrow \infty$, and for all $n \geq 1$ and for all $f \in \mathcal{Q}$

$$
\left\|E\left[f\left(H_{t-1}\right) \mid \mathcal{H}_{t-m-1}\right]-E\left[f\left(H_{t-1}\right)\right]\right\|_{q} \leq c_{t}(f) \zeta_{m}
$$




$$
\left\|f\left(H_{t-1}\right)-E\left[f\left(H_{t-1}\right) \mid \mathcal{H}_{t+m-1}\right]\right\|_{q} \leq c_{t}(f) \zeta_{m+1},
$$

hold for all $t$, and $m \geq 0$.

We shall apply Definition 3 to the class

$$
\mathcal{B}=\left\{f\left(H_{t-1}, x\right)=E\left(\left(Y_{t+h}-\mu\right) 1\left(X_{t} \leq x\right) \mid H_{t-1}\right): x \in \overline{\mathbb{R}}^{p}\right\}
$$

Note that condition (15) is redundant here since $\mathcal{B}$ is a class of $\mathcal{H}_{t-1}$-measurable functions. According to this definition, if $\mathcal{B}$ is an $L^{q}$-mixingale, $q \geq 2$, taking $x=+\infty$ we conclude that $\left(Y_{t}\right)_{t \in \mathbb{Z}}$ is a SMM process.

Hansen (1996) established general conditions for stochastic equicontinuity for smooth (Lipschitz-continuous) mixingale classes of parametric functions. Unfortunately, $v_{n, h}$ involves an indicator function, which is not smooth, and hence, the results in Hansen (1996) are not applicable to the present situation. We shall generalize the stochastic equicontinuity results in Hansen (1996) to non-smooth functions using a martingale approximation and the stochastic equicontinuity theorem for martingales developed in Escanciano (2007). This extension is of independent interest and has applications beyond the present paper.

Define the random function $G_{t}(x):=E\left[\left(Y_{t+h}-\mu\right)^{2} 1\left(X_{t} \leq x\right) \mid \mathcal{H}_{t-1}\right]$. Also, define $\sigma^{2}:=E\left[Y_{t}^{2}\right]$. Define the projection operator $P_{l} W=E\left(W \mid I_{l}\right)-E\left(W \mid I_{l-1}\right)$, for any $W \in L_{2}$. The following regularity conditions are necessary for the subsequent asymptotic analysis.

A1: (a) $\left\{Y_{t}, X_{t}\right\}_{t \in \mathbb{Z}}$ is a strictly stationary and ergodic process such that $\mathcal{B}$ is an $L^{q}$-mixingale class with $c_{t}(f) \equiv c_{t}(x)=C\left\|f\left(I_{t-1}, x\right)\right\|_{s}^{\gamma}$ and $\sum_{m=1}^{\infty} \zeta_{m}<\infty$, for some

$s \geq q$ and $1 / 2 \leq \gamma \leq 1$; (b) $\left|G_{t}\left(x_{1}\right)-G_{t}\left(x_{2}\right)\right| \leq C_{t}\left|x_{1}-x_{2}\right|^{\lambda}$, for each $\left(x_{1}, x_{2}\right) \in$ $\overline{\mathbb{R}}^{p} \times \overline{\mathbb{R}}^{p}$, some $\lambda>0$, and where $C_{t}$ is a $\mathcal{H}_{t-1}$-measurable function; (c) $0<E\left[\left|Y_{t}\right|^{r}\right]<$ $\infty$, with $r=\max (q, 2(1+\delta))$, for some $\delta>0$, and $E\left|C_{t}\right|^{q}<\infty$, for some $q>$ $\max (1,2 p / \lambda)$.

A2: For any $x_{1}, \ldots, x_{m}$ in $\overline{\mathbb{R}}^{p}$ any $\lambda=\left(\lambda_{1}, \ldots, \lambda_{m}\right) \in \mathbb{R}^{p}$ with $|\lambda|=1$ and any $m \in \mathbb{N}$, the sequence $Z_{t, h}=\left(Y_{t+h}-\mu\right) \sum_{j=1}^{m} \lambda_{j} 1\left(X_{t} \leq x_{j}\right)$ satisfies (a) $\left\{Z_{t, h}, \mathcal{F}_{t}\right\}$ is an $L^{2}$-mixingale, and (b) $\sum_{t=-\infty}^{\infty}\left(E\left(P_{0} Z_{t, h}\right)^{2}\right)^{1 / 2}<\infty$.

\section{THEOREM 2:}

(i) Under A1, $v_{n, h}$ is stochastic equicontinuous with respect to $\rho_{m, h}$.

(ii) If in addition A2 holds, then $v_{n, h} \Longrightarrow v_{\infty, h}$, where $v_{\infty, h}$ is a tight Gaussian zero mean process.

Assumption A1 is a mild condition on the DGP and permits a large class of 
nonlinear time series, including heteroskedastic ones. For instance, if $\left(Y_{t}\right)_{t \in \mathbb{Z}}$ is an $L^{q}$-integrable martingale difference sequences (MDS) with respect to $\mathcal{H}_{t}$, then $\mathcal{B}$ satisfies Definition 3. Moreover, as shown in Hansen (1996) a large class of weak dependence processes satisfy Definition 6. Assumption A2 implies the convergence of the finite dimensional distributions.

REMARK: In the case of no-persistence, i.e., when $Y_{t}$ is a MDS with respect to $\mathcal{H}_{t}$, following the proof of our Theorem 2 one can show that the moment assumptions in Theorem 2 can be relaxed to $E\left[\left|Y_{t}\right|^{2(1+\delta)}\right]<\infty$ and $E\left|C_{t}\right|<\infty$.

In the MDS case, the limit process $v_{\infty, h}$ is a Gaussian process in $\ell^{\infty}\left(\overline{\mathbb{R}}^{p}\right)$ with zero-mean, continuous sample paths, and covariance function

$$
K\left(x_{1}, x_{2}\right)=E\left[\left(Y_{t+h}-\mu\right)^{2} 1_{e}\left(X_{t} \leq x_{1}\right) 1_{e}\left(X_{t} \leq x_{2}\right)\right]
$$

Under conditional homoskedasticity and $p=1, v_{\infty, h}$ is distributed as $\sigma^{2} B\left(F_{X}(\cdot)\right)$, where $F_{X}$ is the cdf of $X_{t}$, and $B$ is a standard Brownian Bridge, so the quantiles of norms of $v_{\infty, h}$ are tabulated. For MDS with general heteroskedasticity of unknown form, the asymptotic critical values of norms of $\widehat{\gamma}_{h}$ for testing the significance of $\gamma_{h}$ can be approximated via a wild-type bootstrap approach discussed in the main text.

For the general non-adapted case described in A1, we can apply other resampling methods, such as block-bootstrap or subsampling. In particular, subsampling is a powerful resampling scheme that allows an asymptotically valid inference under very general conditions on the DGP, see the monograph by Politis, Romano and Wolf (1999). With an abuse of notation we write $\Gamma\left(v_{n, h, w}\right)=\left\|v_{n, h, w}\right\|_{d}$ as a function of the data $\left\{Z_{t} \equiv\left(Y_{t+h}, X_{t}^{\prime}\right)^{\prime}\right\}_{t \in \mathbb{Z}}, \Gamma\left(v_{n, h, w}\right)=\Gamma\left(v_{n, h, w}\left(Z_{1}, \ldots, Z_{n}\right)\right)$. Let $G_{n}^{\Gamma}(w)$ be the cdf

$$
G_{n}^{\Gamma}(z)=P\left(\Gamma\left(v_{n, h, w}\right) \leq z\right)
$$

Let $\Gamma\left(v_{n, h, w}^{b, i}\right)=\Gamma\left(v_{n, h, w}\left(Z_{i}, \ldots, Z_{i+b-1}\right)\right)$ be the norm computed with the subsample $\left(Z_{i}, \ldots, Z_{i+b-1}\right)$ of size $b$. We note that each subsample of size $b$ (taken without replacement from the original data) is indeed a sample of size $b$ from the true DGP. Hence, it is clear that one can approximate the sampling distribution $G_{n}^{\Gamma}(z)$ using the distribution of the values of $\Gamma\left(v_{n, h, w}^{b, i}\right)$ computed over the $n-b+1$ different subsamples of size $b$. That is, we approximate $G_{n}^{\Gamma}(z)$ by

$$
G_{n, b}^{\Gamma}(z)=\frac{1}{n-b+1} \sum_{i=1}^{n-b+1} 1\left(\Gamma\left(v_{n, h, w}^{b, i}\right) \leq z\right) \quad z \in[0, \infty)
$$


Let $c_{n, 1-\tau, b}^{\Gamma}$ be the $(1-\tau)$-th sample quantile of $G_{n, b}^{\Gamma}(z)$, i.e.,

$$
c_{n, 1-\tau, b}^{\Gamma}=\inf \left\{z: G_{n, b}^{\Gamma}(z) \geq 1-\tau\right\}
$$

The theoretical justification of the subsampling approximation can be grounded on Theorem 3.5.1 in Politis, Romano and Wolf (1999) under mild conditions on the strong mixing coefficients and the parameter $b$. With these asymptotic results one can compute (uniform) confidence bands for the measures $\gamma_{h}(\cdot)$.

\subsubsection{Asymptotic theory under strong mixing conditions}

In this subsection we establish the stochastic equicontinuity of $\omega_{n, h}$ under strong mixing assumptions. This will follow from the general results in Andrews and Pollard (1994). We require the following assumption.

B1: (a) $\left\{Y_{t}, X_{t}\right\}_{t \in \mathbb{Z}}$ is a strictly stationary; (b) $\left|F_{X}\left(x_{1}\right)-F_{X}\left(x_{2}\right)\right| \leq C\left|x_{1}-x_{2}\right|^{2 \lambda_{1}}$, for each $\left(x_{1}, x_{2}\right) \in \overline{\mathbb{R}}^{p} \times \overline{\mathbb{R}}^{p}$, some $\lambda_{1}>0$; (c) $\left|F_{Y}\left(y_{1}\right)-F_{Y}\left(y_{2}\right)\right| \leq C\left|y_{1}-y_{2}\right|^{2 \lambda_{2}}$, for each $\left(y_{1}, y_{2}\right) \in \overline{\mathbb{R}} \times \overline{\mathbb{R}}$, some $\lambda_{2}>0$ (c) $\left\{Y_{t}, X_{t}\right\}_{t \in \mathbb{Z}}$ is a strong mixing process with mixing coefficients such that $\sum_{h=1}^{\infty} h^{Q-2} \alpha_{h}^{\gamma /(Q+\gamma)}<\infty$, for some even integer $Q \geq 2$, such that $Q>\left(\frac{2+\gamma}{2}\right) \frac{p+1}{\lambda}$, some $\gamma>0$ and $\lambda=\min \left(\lambda_{1}, \lambda_{2}\right)$.

Theorem 3: Under B1, $\omega_{n, h}$ is stochastic equicontinuous with respect to $\rho_{d, h}$.

Different assumptions to B1 are also possible using other mixing concepts under the sup-metric; see, e.g., Rio (2000) and Andrews (1993, p. 200). For strong mixing sequences Theorem 2.17 in Bosq (2000) gives the asymptotic distribution for $L_{2}$-norms under the mild condition $\sum_{h=1}^{\infty} \alpha_{h}<\infty$. See also Bradley (2007) for other asymptotic results under mixing.

For distributional dependence the least persistent case corresponds to that where $Y_{t+h}$ is independent of $\mathcal{F}_{t}$. When $p=1$, the limit process of $\omega_{n, h}$, say $\omega_{\infty, h}$, is distributed as $W\left(F_{X}(\cdot), F_{Y}(\cdot)\right)$ where $W(u, v)$ is a standard bivariate Brownian Bridge, and therefore, continuous functionals of $\widehat{\eta}_{h}$ are asymptotic distribution free, with critical values that can be tabulated. For $p>1$, we can use a wild-bootstrap type procedure as for $\widehat{\gamma}_{h}$. That is, we approximate the distribution of $\widehat{\eta}_{h}$ with that of

$$
\widehat{\eta}_{h}^{*}(x)=\frac{1}{n-h} \sum_{t=1}^{n-h} 1_{c, Y_{t+h}}(y) 1_{c, X_{t}}(x) V_{t}
$$

Of course, other alternative bootstrap procedures are available, e.g., the classical bootstrap of Efron (1979). For the more general case described in B1, a subsampling 
approximation, as discussed in Section 4.1 is, of course, possible.

\subsection{Mathematical Proofs}

Proof of Theorem 1. First, by simple application of the Cauchy-Swartz's inequality, a SMM process satisfies $\lim _{h \rightarrow \infty}\left\|\gamma_{t, h}\right\|_{\infty}=0$. The reciprocal is more involved. From $\sup _{t} E Y_{t}^{2}<C$, we have that the sequence $\left(Y_{t+h}\right)_{h \in \mathbb{N}}$ is uniformly tight. Also, from Prohorov's theorem, we have that any subsequence of $\left(Y_{t+h}\right)_{h \in \mathbb{N}}$ has a further subsequence $\left(Y_{t+h_{s}}\right)_{s \in \mathbb{N}}$ which converges in distribution to a r.v. $Y$, see Billingsley (1968). Because the subsequence is uniform integrable, the convergence is also in $L_{2}(\mathbb{R}, F)$. From Proposition 1.2 in Chung and Williams (1990) $m_{t, h_{s}}$ also converges to $E\left(Y \mid X_{t}\right)$ in $L_{2}(\mathbb{R}, F)$. Then

$$
\left\|\gamma_{t, h_{s}}\right\|_{\infty}^{2} \rightarrow\left\|E\left[(Y-\mu) 1\left(X_{t} \leq \cdot\right)\right]\right\|_{\infty}^{2} \quad \text { as } h_{s} \rightarrow \infty
$$

From $\lim _{h \rightarrow \infty}\left\|\gamma_{t, h}\right\|_{\infty}=0$ we have that $E\left(Y \mid X_{t}\right)=\mu$ a.s. Therefore, from the uniqueness of the limit, we have that $\left(m_{t, h}\right)_{h \in \mathbb{N}}$ converges in $L_{2}(\mathbb{R}, F)$ to $\mu$ and the process is SMM.

In the proof of Theorem 2 we use extensively the following weak convergence theorem, which is a corollary of a general result in Escanciano (2007). Define the process

$$
R_{n}(x):=\frac{1}{\sqrt{n}} \sum_{t=1}^{n} Z_{t} 1_{e}\left(X_{t} \leq x\right), \quad x \in \overline{\mathbb{R}}^{p} .
$$

Let $F$ be the cumulative distribution function (cdf) of $X_{t}$. We define the random function $G_{t}(x):=E\left[Z_{t}^{2} 1\left(X_{t} \leq x\right) \mid \mathcal{H}_{t-1}\right]$, where $\mathcal{H}_{t}$ is the $\sigma$-field generated by $\left(Z_{t}, X_{t}, Z_{t-1}, X_{t-1}, \ldots\right)$. The following regularity condition is necessary for the subsequent asymptotic analysis. Define the pseudometric, with $s \geq 2$,

$$
\rho_{s}\left(x_{1}, x_{2}\right)=\left(E\left[\left|Z_{t}\right|^{s}\left|1_{e}\left(X_{t} \leq x_{1}\right)-1_{e}\left(X_{t} \leq x_{2}\right)\right|^{s}\right]\right)^{1 / s}
$$

C1: (a) $\left\{Z_{t}\right\}_{t \in \mathbb{Z}}$ is a strictly stationary and ergodic MDS with respect to $\mathcal{H}_{t}$, with $E\left[\left|Z_{t}\right|^{s}\right]<\infty$ for some $s \geq 2$; (b) $\left|G_{t}\left(x_{1}\right)-G_{t}\left(x_{2}\right)\right| \leq C_{t}\left|x_{1}-x_{2}\right|^{\lambda}$, for each $\left(x_{1}, x_{2}\right) \in$ $\overline{\mathbb{R}}^{p} \times \overline{\mathbb{R}}^{p}$, some $\lambda>0$, and where $C_{t}$ is a stationary sequence with $E\left|C_{t}\right|<\infty$; (c) $F$ is absolutely continuous.

TheOREM A1: Under $C 1, R_{n}$ is stochastically equicontinuous with respect to $\rho_{s}$. 
Proof of Theorem A1. It follows from a routine extension to multivariate regressors of Theorem 1 in Escanciano and Mayoral (2010).

Proof of Theorem 2. Defining the projector operator $P_{l} W=E\left(W \mid H_{l}\right)-$ $E\left(W \mid H_{l-1}\right), W \in L_{2}$, we can show that, uniformly in $x$,

$$
\begin{aligned}
v_{n, h}(\cdot)= & \sqrt{n-h}\left(\widehat{\gamma}_{h}(\cdot)-\gamma_{h}(\cdot)\right) \\
= & \sum_{j=1}^{h+1} \frac{1}{\sqrt{n-h}} \sum_{t=1}^{n} P_{t+j-1}\left\{\left(Y_{t+h}-\mu\right) 1_{e}\left(X_{t} \leq x\right)\right\} \\
& +\frac{1}{\sqrt{n-h}} \sum_{t=1}^{n} E\left(\left(Y_{t+h}-\mu\right) 1_{e}\left(X_{t} \leq x\right) \mid H_{t-1}\right)-\gamma_{h}(\cdot)+o_{P}(1) \\
\equiv & \sum_{j=1}^{h+1} A_{n j}(x)+B_{n}(x)+o_{p}(1) .
\end{aligned}
$$

Note that $A_{n j}$ are martingales, so results to establish the stochastic equicontinuity of $A_{n j}$, such as our previous Theorem A1 or Theorem 1 in Escanciano (2007) are applicable. Note that for $j \geq 2$,

$$
A_{n j}(x)=\frac{1}{\sqrt{n-h}} \sum_{t=1}^{n}\left\{P_{t+j-1} Y_{t+h}\right\} 1_{e}\left(X_{t} \leq x\right),
$$

so Theorem A1 applies to $Z_{t}=P_{t+j-1} Y_{t+h}$, after noting that, for $s \geq 2$,

$$
\begin{aligned}
E\left[\left|Z_{t}\right|^{s}\right] & \leq E\left[\left|Y_{t+h}-E\left(Y_{t+h} \mid H_{t+j-2}\right)\right|^{s}\right] \\
& \leq E\left[\left|Y_{t+h}\right|^{s}\right]<\infty
\end{aligned}
$$

The stochastic equicontinuity of $A_{n 1}$ follows from the following arguments. Let $B_{k}=$ $\left[x_{k}, y_{k}\right]$, for $k=1, \ldots, N_{\varepsilon}$, a partition of $\overline{\mathbb{R}}^{p}$ in $\varepsilon$-brackets with respect to $\rho_{m, h}$. With an abuse of notation, we denote the previous partition associated to $\varepsilon=2^{-q}$ simply by $\mathcal{B}_{q}=\left\{B_{q k} ; 1 \leq k \leq N_{q}\right\}$. Without loss of generality we can assume that the finite partitions described before are nested. It is clear that

$$
\sum_{q=1}^{\infty} 2^{-q} \sqrt{\log N_{q}}<\infty
$$

which readily verifies condition (5) in Escanciano (2007). It remains to verify condition (6) in that paper. From the monotonicity of $1\left(X_{t} \leq x\right), \mathrm{A} 1(\mathrm{~b})$ and keeping the 
notation in Escanciano (2007), $\alpha_{n}\left(\mathcal{B}_{q}\right)$ is defined as

$$
\begin{aligned}
& \max _{1 \leq k \leq N_{q}}\left|\frac{1}{n} \sum_{t=1}^{n} E\left[\sup _{x, y \in B_{q k}}\left|P_{t}\left\{\left(Y_{t+h}-\mu\right) 1_{e}\left(X_{t} \leq x\right)\right\}-P_{t}\left\{\left(Y_{t+h}-\mu\right) 1_{e}\left(X_{t} \leq y\right)\right\}\right|^{2} \mid \mathcal{H}_{t-1}\right]\right| \\
\leq & C \max _{1 \leq k \leq N_{q}}\left|n^{-1} \sum_{t=1}^{n} E\left[\left(Y_{t+h}-\mu\right)^{2}\left|1_{e}\left(x_{k} \leq X_{t} \leq y_{k}\right)\right| \mid \mathcal{H}_{t-1}\right]\right| \leq O_{P}\left(2^{-2 q}\right) .
\end{aligned}
$$

This proves (6) in Escanciano (2007), and hence the stochastic equicontinuity of $A_{n 1}$.

As for $B_{n}(x)$, we shall apply Theorem 1 in Hansen (1996). First note that the class of functions $\left\{f\left(H_{t-1}, x\right)=E\left(\left(Y_{t+h}-\mu\right) 1\left(X_{t} \leq x\right) \mid H_{t-1}\right): x \in \overline{\mathbb{R}}^{p}\right\}$ satisfies the Lipschitz condition

$$
\left|f\left(H_{t-1}, x\right)-f\left(H_{t-1}, x\right)\right| \leq C_{t}^{1 / 2}\left|x_{1}-x_{2}\right|^{\lambda / 2}
$$

Therefore, the class $\mathcal{B}$ satisfies the conditions of Theorem 1 in Hansen (1996), and the stochastic equicontinuity of $B_{n}$ follows. The finite sum of stochastic equicontinuous processes is stochastic equicontinuous. Finally, the convergence of the finite dimensional distributions in (ii) follows directly from A2 and Corollary 1 in Dedecker, Merlevede and Volny (2007).

Proof of Theorem 3. The theorem follows from a direct application of Theorem 2.2 in Andrews and Pollard (1994).

\section{REFERENCES}

Acharya, V.V., L. Pedersen, T. Philippon, and M. Richardson, 2017, Measuring Systemic Risk. The Review of Financial Studies 30, 2-47.

Andrews, D. W. K., 1993, An introduction to econometric applications of empirical process theory for dependent random variables. Econometric Reviews 12, 183-216.

Andrews, D. W. K., 1994, Empirical process methods in econometrics. In Handbook of Econometrics, Volume 4, ed. by R.F. Engle and D. McFadden. New York: NorthHolland, 2247-2294.

Andrews, D. W. K. and Pollard, D., 1994, An introduction to functional central limit theorems for dependent stochastic processes. International Statistical Review 62, 119-132. 
Ang, A. and Bekaert, G. 2002, International Asset Allocation with Regime Shifts. Review of Financial Studies 15, 1137-1187.

Ang, A. and Chen, J., 2002, Asymmetric correlations of equity portfolios. Journal of Financial Econometrics 63, 443-494.

Ango Nze, P. and Doukhan, P., 2004, Weak dependence: models and applications to econometrics. Econometric Theory 20, 995-1045.

Auestad, B. and Tjøstheim, D., 1990, Identification of nonlinear time series: First order characterization and order determination. Biometrika 77, 669-687.

Bradley, R.C., 2007. Introduction to Strong Mixing Conditions, Vols. 1, 2, and 3. Kendrick Press, Heber City (Utah).

Bae, K.H., Karolyi, G.A. and Stulz, R.M., 2003, A New Approach to Measuring Financial Contagion. The Review of Financial Studies, 16, 717-763.

Bansal, R. and Yaron, A., 2004, Risks for the long run: a potential resolution of asset pricing puzzles. The Journal of Finance, 59, 1481-1509.

Bierens, H.J., 1982, Consistent model specification tests. Journal of Econometrics 20, $105-134$.

Billingsley, P., 1968, Convergence of Probability Measures. Wiley, New York.

Bollerslev, T. and Engle, R.F., 1993, Common persistence in conditional variances. Econometrica, 61, 167-186.

Bosq, D., 2000, Linear processes in function spaces. Theory and applications. Lecture Notes in Statistics 149 (Springer).

Buja, A., 1990, Remarks on functional canonical variates, alternating least squares methods, and ACE. The Annals of Statistics 18, 1032-1069.

Christoffersen, P., Errunza, V., Jacobs, K. and Langlois, H., 2012, Is the Potential for International Diversification Disappearing? A Dynamic Copula Approach. The Review of Financial Studies 25, 3711-3751.

Chung, K.L. and Williams, R.J., 1990, Introduction to stochatic integration. Birkhauser, Boston.

Clarida, R. H. and Taylor, M. P., 2003, Nonlinear permanent-temporary decompositions in macroeconomics and finance. Economic Journal 113, 125-139.

Davidson, J., 1994, Stochastic limit theory. Oxford: Oxford University Press.

de Jong, R., 1997, Central limit theorems for dependent heterogeneous random variables. Econometric Theory 13, 353-367.

Dedecker, J., Merlevede, F. and Volny, D., 2007, On the weak invariance principle for 
non-adapted sequences under projective criteria. Journal of Theoretical Probability 20, 971-1004.

Dette, H., Hallin, M., Kley, T. and Volgushev, S., 2015, Of copulas, quantiles, ranks and spectra: an $L_{1}$-approach to spectral analysis. Bernoulli 21, 781-831.

Efron, B., 1979, Bootstrap methods: another look at the jacknife. The Annals of Statistics 7, 1-26.

Engle, R.F. and Bollerslev, T., 1986, Modelling the persistence of conditional variances. Econometric Reviews 5, 81-87.

Escanciano, J. C., 2006, Goodness-of-fit tests for linear and non-linear time series models. Journal of the American Statistical Association 101, 531-541.

Escanciano, J. C., 2007, Model checks using residual marked empirical processes. Statistica Sinica 17, 115-138.

Escanciano J.C. and Lobato I.N., 2009, An automatic portmanteau test for serial correlation. Journal of Econometrics 151, 140-149.

Escanciano, J.C. and Mayoral, S., 2010, Data-driven smooth tests for the martingale difference hypothesis. Computational Statistics \& Data Analysis, 54, 1983-1998.

Escanciano, J. C. and Velasco, C., 2006a, Testing the martingale difference hypothesis using integrated regression functions. Computational Statistics \& Data Analysis 51, 2278-2294.

Escanciano, J. C., and Velasco, C., 2006b, Generalized spectral tests for the martingale difference hypothesis. Journal of Econometrics 134, 151-185.

Forbes, K. and Rigobon, R. 2002, No Contagion, Only Interdependence: Measuring Stoock Market Co-Movements. Journal of Finance 57, 2223-2261.

Francq, C. J.M. Zakoian, 2010, GARCH Models: Structure, Statistical Inference and Financial Applications. Wiley, Chichester, UK.

Gallant, A. R., Rossi, P. E. and Tauchen, G., 1993, Nonlinear dymanics structures. Econometrica 61, 871-907.

Gourieroux, C. and Jasiak, J., 1999, Nonlinear persistence and copersistence. Preprint, Institut National de la Statistique et des Etudes Economiques.

Gourieroux, C. and Jasiak, J., 2000, Nonlinear innovations and impulse responses. Preprint, Institut National de la Statistique et des Etudes Economiques.

Gourieroux, C. and Jasiak, J., 2002, Nonlinear autocorrelograms: an application to inter-trade durations. Journal of Time Series Analysis 23, 127-154.

Granger, C. W. J., 1995, Modelling nonlinear relationships between extended-memory 
variables. Econometrica 63, 265-279.

Granger, C. W. J., 2003, Time series concepts for conditional distributions. Oxford Bulletin of Economics and Statistics 65 (supplement), 639-701.

Granger, C. W. J. and Hallman, J., 1991, Nonlinear transformations of integrated time series. Journal of Time Series Analysis 12, 207-224.

Granger, C. W. J., Maasoumi, E. and Racine, J., 2004, A dependence metric for possibly nonlinear processes. Journal of Time Series Analysis 25, 649-669.

Hansen, B., 1996, Stochastic equicontinuity for unbounded dependent heterogeneous arrays. Econometric Theory 12, 347-359.

Hartmann, P., Straetmans, S. and de Vries, C.G. 2004, Asset Market Linkages in Crisis Periods. The Review of Economics and Statistics, 86, 313-326.

Hoeffding, W. 1940, Scale-invariant correlation theory. In Fisher, N.I, Sen, P.K (Eds), The Collective Works of Wassily Hoeffding. Springer-Verlag, New York, 1994.

Hong, Y., 1999, Hypothesis Testing in Time Series via the Empirical Characteristic Function. Journal of American Statistical Association, 84, 1201-1220.

Kapetanios, G., 2007, Measuring conditional persistence in nonlinear time series. Oxford Bulletin of Economics and Statistics 69, 363-386.

King, M.A. and Wadhwani, S. 1990, Transmission of Volatility Between Stock Markets. The Review of Financial Studies, 3, 5-33.

Koop, G., Pesaran, M. H. and Potter, S. M., 1996, Impulse response analysis in nonlinear multivariate models. Journal of Econometrics 74, 119-147.

Koul, H. L. and Stute, W., 1999, Nonparametric model checks for time series. The Annals of Statistics 27, 204-236.

Lin, W.L., Engle, R. and Ito, T. 1994, Do Bulls and Bears Move Across Borders? International Transmission of Stock Returns and Volatility. Review of Financial Studies $7,507-538$.

Lo, A.W. and MacKinlay, A.C., 1999, A non-random walk down Wall street. Princeton. Longin, F. and Solnik, B., 2001, Extreme correlation of international equity markets. Journal of Finance 56 (2), 649-676.

Malevergne, Y. and Sornette, D. 2006, Extreme Financial Risks. From Dependence to Risk Management. Springer Science \& Business Media.

McDonald, D.J., Shalizi, C.R and Schervish, M. 2015, Estimating Beta-Mixing Coefficients vis Histograms. Electronic Journal of Statitics, 9, 2855-2883.

McLeish, D. L., 1974, Dependent central limit theorems and invariance principles. The 
Annals of Probability 24, 620-628.

Morgenstern, O. 1959, International Financial Transactions and the Business Cycle. Princeton, NJ: Princeton University Press.

Politis, D. N., Romano, J.P. and Wolf, M., 1999, Subsampling. Springer-Verlag, New York.

Poterba, J.M. and Summers, L.H., 1986, The persistence of volatility and stock market fluctuations. The American Economic Review 76, 1142-1251.

Rio, E., 2000, Theorie asymptotique des processus aleatoires faiblement dependants. Math'ematiques et Applications 31. Springer, Berlin

Robinson, P.M., 1983, Nonparametric estimators for time series. Journal of Time Series Analysis 4, 185-207.

Sims, C., 1980, Macroeconomics and reality. Econometrica 48, 1-48.

Stute, W., 1997, Nonparametic model checks for regression. The Annals of Statistics $25,613-641$.

Terasvirta, T., Tjostheim, D. and Granger, C.W.J,. 2011, A Review of Modelling Nonlinear Economic Time Series. Oxford: Oxford University Press.

van der Vaart, A. W. and Wellner, J. A., 1996, Weak convergence and empirical processes. Springer, New York.

Wu, C.F.J., 1986, Jacknife, Bootstrap and other resampling methods in regression analysis (with discussion). The Annals of Statistics 14, 1261-1350. 
TABLES

Table 1. Descriptive Statistics: Stock Indices

\begin{tabular}{ccccccc}
\hline \hline Statistic & SP500 & DJ & FTSE & DAX & HANG & NIK \\
\hline \hline Mean & 0.0071 & 0.0066 & 0.0008 & 0.0066 & 0.0003 & -0.0019 \\
Std & 0.5854 & 0.5370 & 0.5624 & 0.6499 & 0.7247 & 0.7234 \\
Kurtosis & 12.4924 & 12.4453 & 9.8018 & 8.3768 & 11.1500 & 10.3124 \\
Median & 0.0296 & 0.0232 & 0.0150 & 0.0331 & 0.0000 & 0.0205 \\
Min & -4.1126 & -3.5614 & -4.0235 & -3.2283 & -5.8986 & -5.2597 \\
Max & 4.7587 & 4.5637 & 4.0755 & 4.6893 & 5.8225 & 5.7477 \\
Corr(1) & -0.1035 & -0.1043 & -0.0338 & 0.0062 & -0.0285 & -0.0475 \\
DBP-pval & 0.0000 & 0.0000 & 0.0976 & 0.7615 & 0.1642 & 0.0215 \\
\hline \hline
\end{tabular}

Note: DBP-pval denotes the p-value of the Data Driven Box Pierce test of Escanciano and Lobato (2009) for testing serial correlation.

Table 2. Bootstrap P-value at $h=1$ : Mean Dependence

\begin{tabular}{ccccccc}
\hline \hline$Y_{t+1} / X_{t}$ & SP500 & DJ & FTSE & DAX & HANG & NIK \\
\hline \hline SP500 & 0.0067 & 0.0067 & 0.0000 & 0.2767 & 0.6400 & 0.5400 \\
DJ & 0.0067 & 0.0033 & 0.0000 & 0.3167 & 0.6767 & 0.5667 \\
FTSE & 0.2167 & 0.2567 & 0.5967 & 0.0567 & 0.1200 & 0.7367 \\
DAX & 0.0000 & 0.0000 & 0.0000 & 0.3067 & 0.4633 & 0.1967 \\
HANG & 0.0000 & 0.0000 & 0.0000 & 0.0000 & 0.5033 & 0.3267 \\
NIK & 0.0000 & 0.0000 & 0.0000 & 0.0000 & 0.1233 & 0.2833 \\
\hline \hline
\end{tabular}

Note: Bootstrap p-values for $K S_{n}(h)$ at $h=1$.

Table 3. Bootstrap P-Value at $h=1$ : Tail Risk

\begin{tabular}{ccccccc}
\hline \hline$Y_{t+1} / X_{t}$ & SP500 & DJ & FTSE & DAX & HANG & NIK \\
\hline \hline SP500 & 0.0367 & 0.1100 & 0.0000 & 0.6567 & 0.8467 & 0.9600 \\
DJ & 0.0467 & 0.1033 & 0.0000 & 0.6067 & 0.7367 & 0.9833 \\
FTSE & 0.1567 & 0.1633 & 0.2500 & 0.9233 & 0.4233 & 0.8400 \\
DAX & 0.0467 & 0.0267 & 0.0000 & 0.5267 & 0.3333 & 0.7833 \\
HANG & 0.0000 & 0.0000 & 0.0000 & 0.1133 & 0.4667 & 0.4500 \\
NIK & 0.0033 & 0.0067 & 0.0000 & 0.0333 & 0.7400 & 0.0933 \\
\hline \hline
\end{tabular}

Note: Bootstrap p-values for $S T R_{n}(h)$ at $\mathcal{T}=\left\{x \in \mathbb{R}: \hat{F}_{h}^{-1}(0.01) \leq x \leq \hat{F}_{h}^{-1}(0.05)\right\}$ at $h=1$. 
TABle 4 . Bootstrap P-VAlue at $h=1$ : MES

\begin{tabular}{ccccccc}
\hline \hline$Y_{t+1} / X_{t}$ & SP500 & DJ & FTSE & DAX & HANG & NIK \\
\hline \hline SP500 & 0.4333 & 0.4633 & 0.0000 & 0.5733 & 0.4233 & 0.7500 \\
DJ & 0.5200 & 0.5533 & 0.0000 & 0.4733 & 0.4167 & 0.7867 \\
FTSE & 0.5167 & 0.4800 & 0.4333 & 0.6167 & 0.0100 & 0.2300 \\
DAX & 0.0000 & 0.0000 & 0.0000 & 0.8800 & 0.7367 & 0.3600 \\
HANG & 0.0000 & 0.0000 & 0.0000 & 0.0000 & 0.7400 & 0.3367 \\
NIK & 0.0000 & 0.0000 & 0.0000 & 0.0000 & 0.9433 & 0.0833 \\
\hline \hline
\end{tabular}

Note: Bootstrap p-values for $\widehat{S E S}_{h}(x)$ at $x=\hat{F}_{h}^{-1}(0.05)$ at $h=1$.

Table 5. Rejections Lags 1-300: volatility

\begin{tabular}{ccccccc}
\hline \hline$Y_{t+1}^{2} / X_{t}$ & SP500 & DJ & FTSE & DAX & HANG & NIK \\
\hline \hline SP500 & 102 & 105 & 130 & 60 & 149 & 61 \\
DJ & 95 & 95 & 122 & 60 & 145 & 64 \\
FTSE & 79 & 82 & 95 & 44 & 117 & 51 \\
DAX & 84 & 86 & 92 & 56 & 86 & 40 \\
HANG & 94 & 86 & 100 & 46 & 120 & 49 \\
NIK & 53 & 46 & 62 & 35 & 69 & 39 \\
\hline \hline
\end{tabular}

FigURES
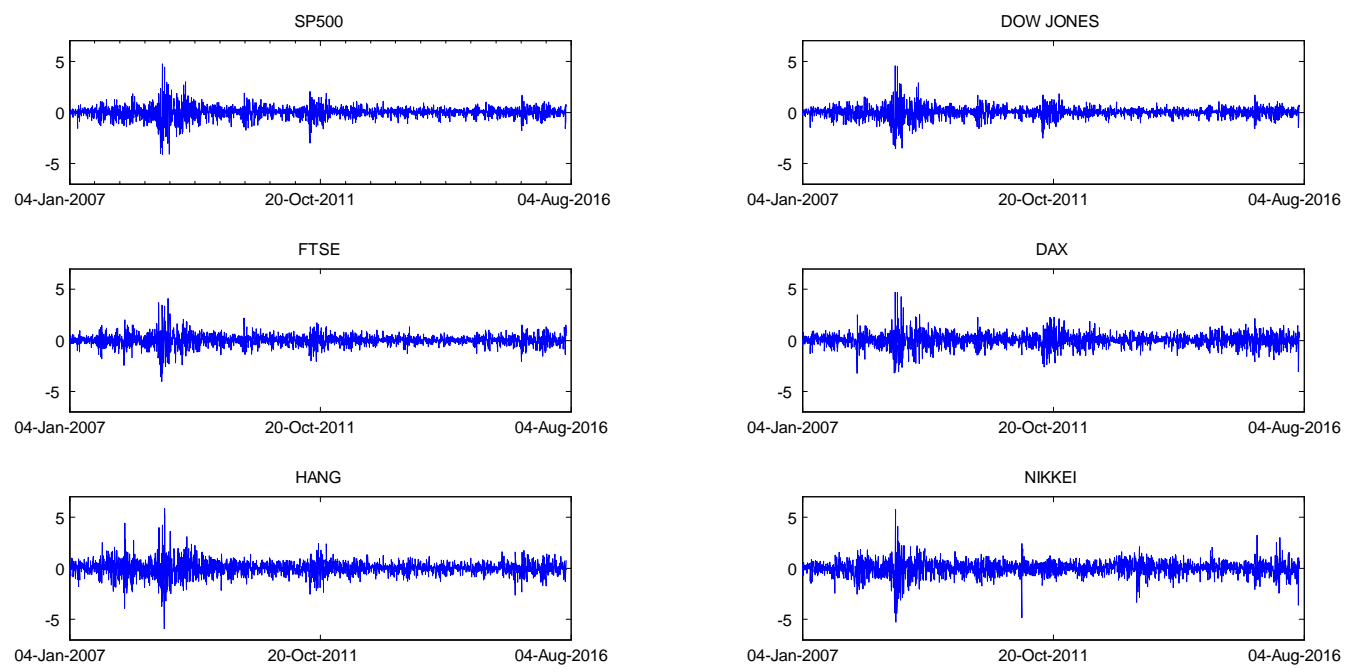

Figure 1: Daily stock returns from Jan 4, 2007 until Jun 30, 2016. 

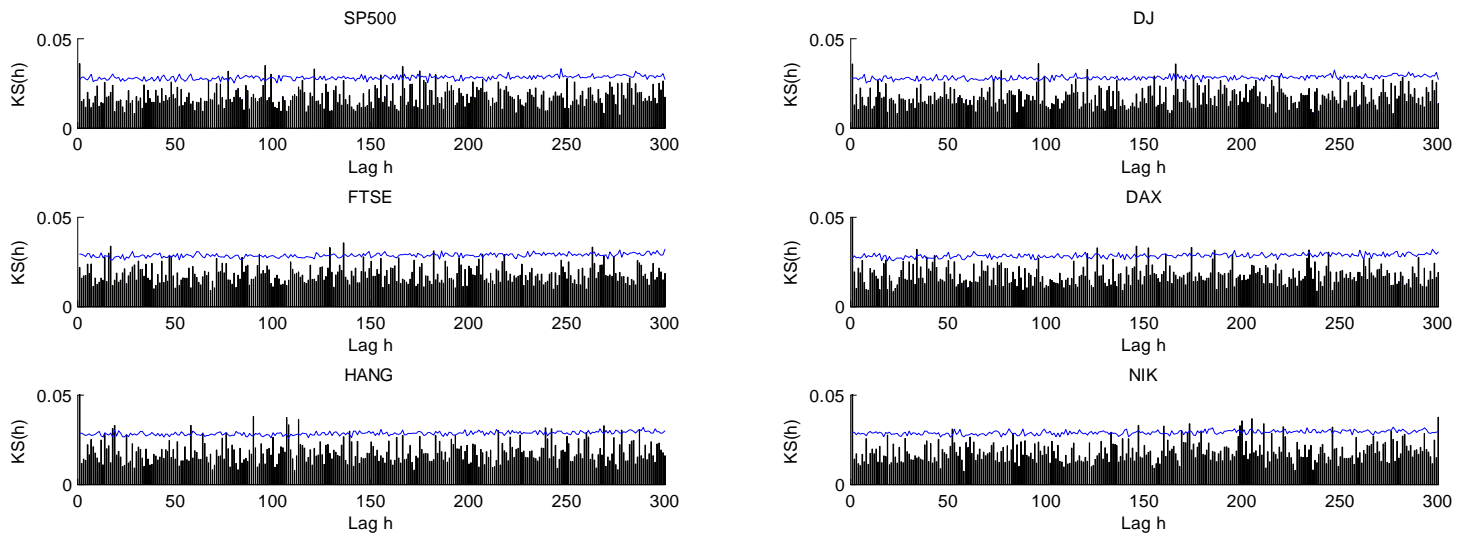

Figure 2: NAC for Mean Dependence with SP500 as conditioning stock. 95\% Bootstrap confidence intervals for significance testing.
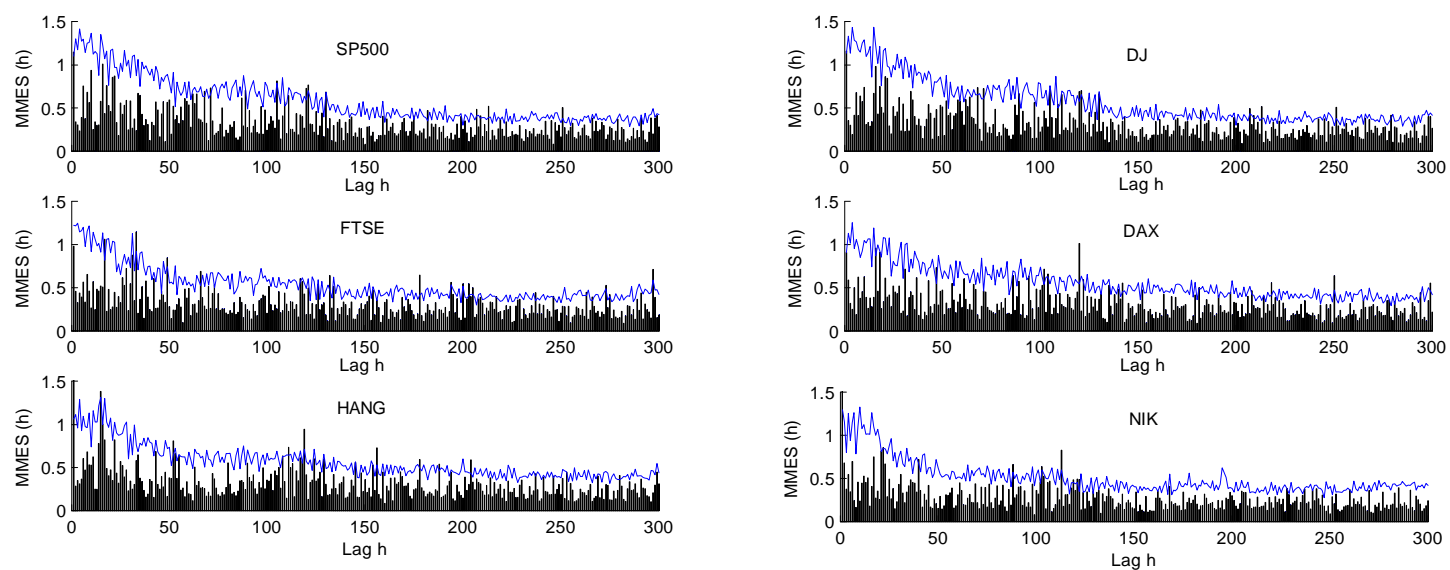

Figure 3: MMES plot for Tail Dependence at quantiles 1\%-5\% with SP500 as conditioning stock. 95\% Bootstrap confidence intervals for significance testing. 

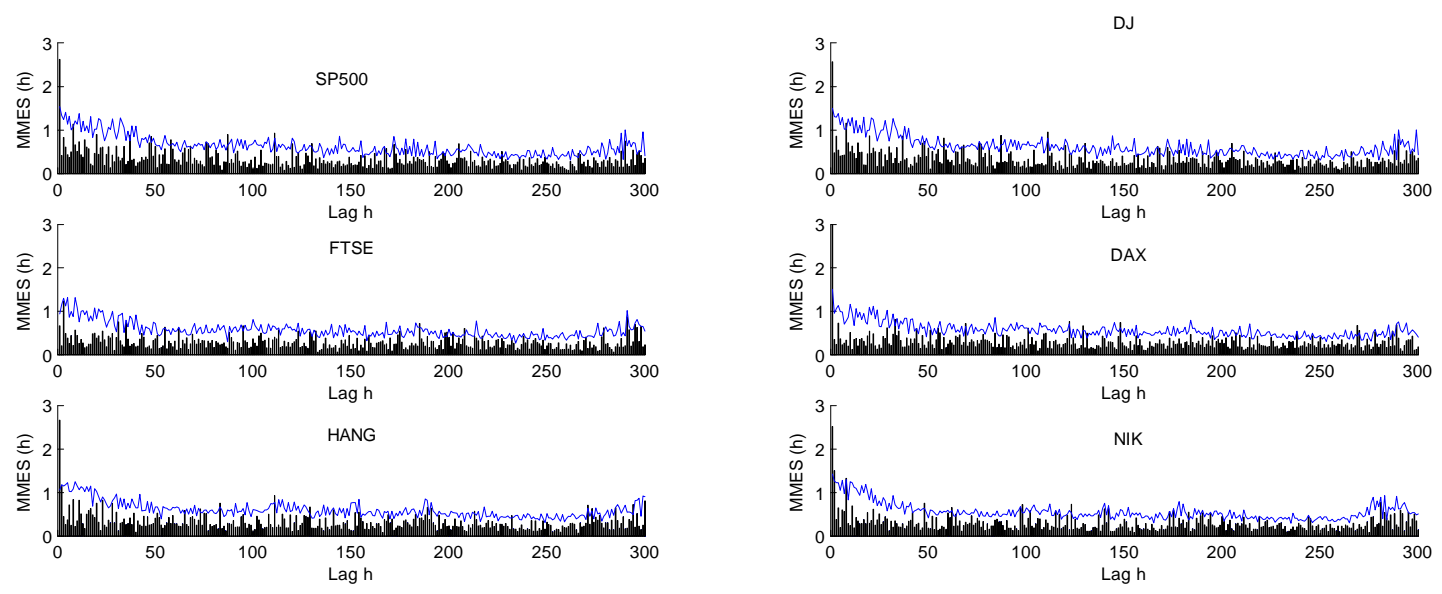

Figure 4: MMES plot for Tail Dependence at quantiles 1\%-5\% with FTSE as conditioning stock. 95\% Bootstrap confidence intervals for significance testing.
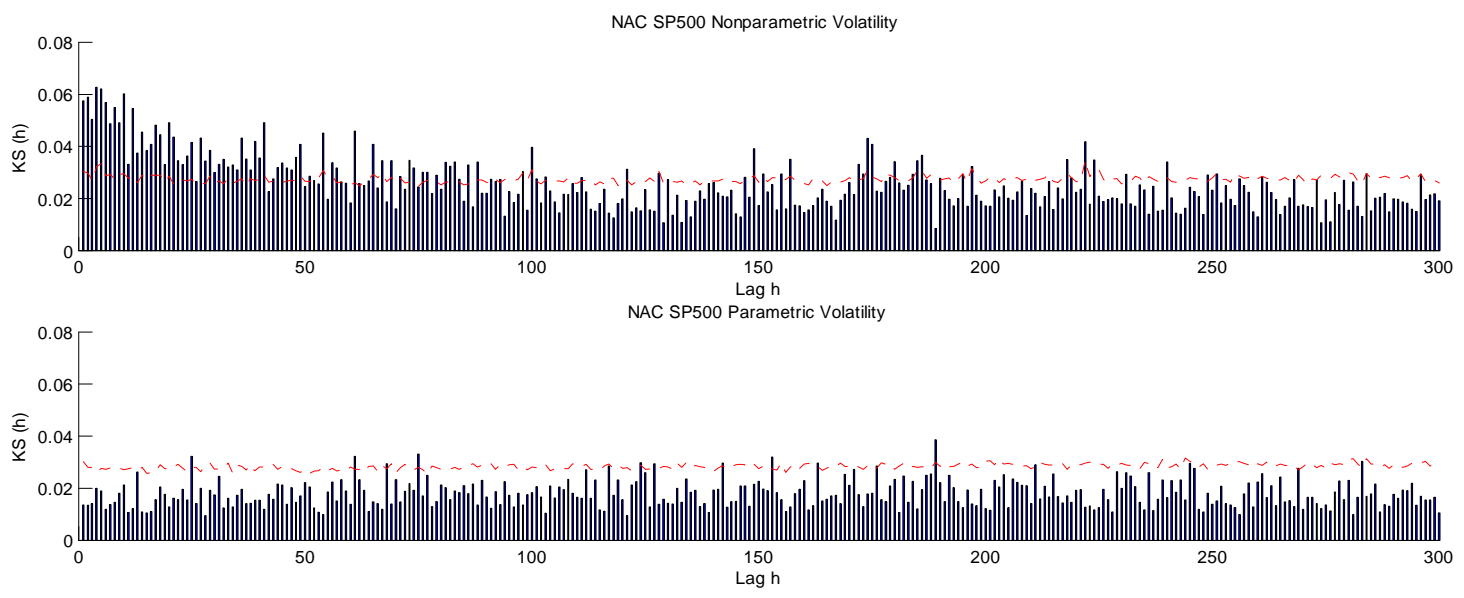

FIgURE 5: Upper plot: NAC for volatility measures based on SP500 data. Lower plot: NAC for volatility measures based on simulated data from a AR(1)-GARCH(1,1) model with Student-t innovations with parameters obtained from fitting the SP500 data. 

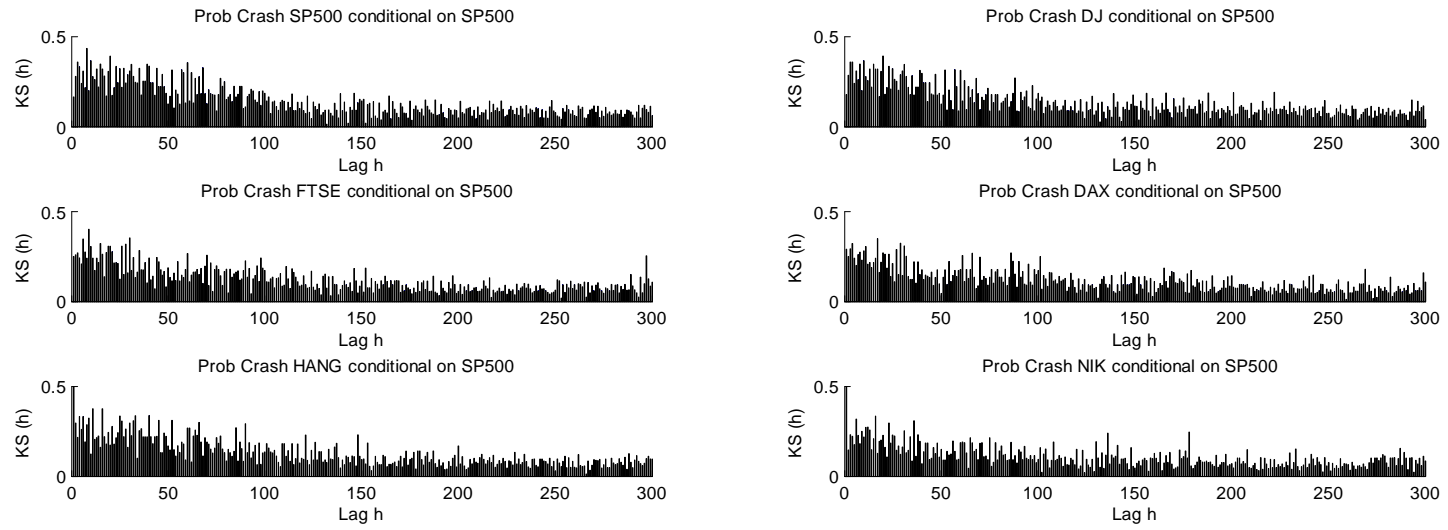

FiguRE 6: Estimated maximal conditional probabilities of crashes conditioning on SP500 for quantiles $1 \%$ to $5 \%$.
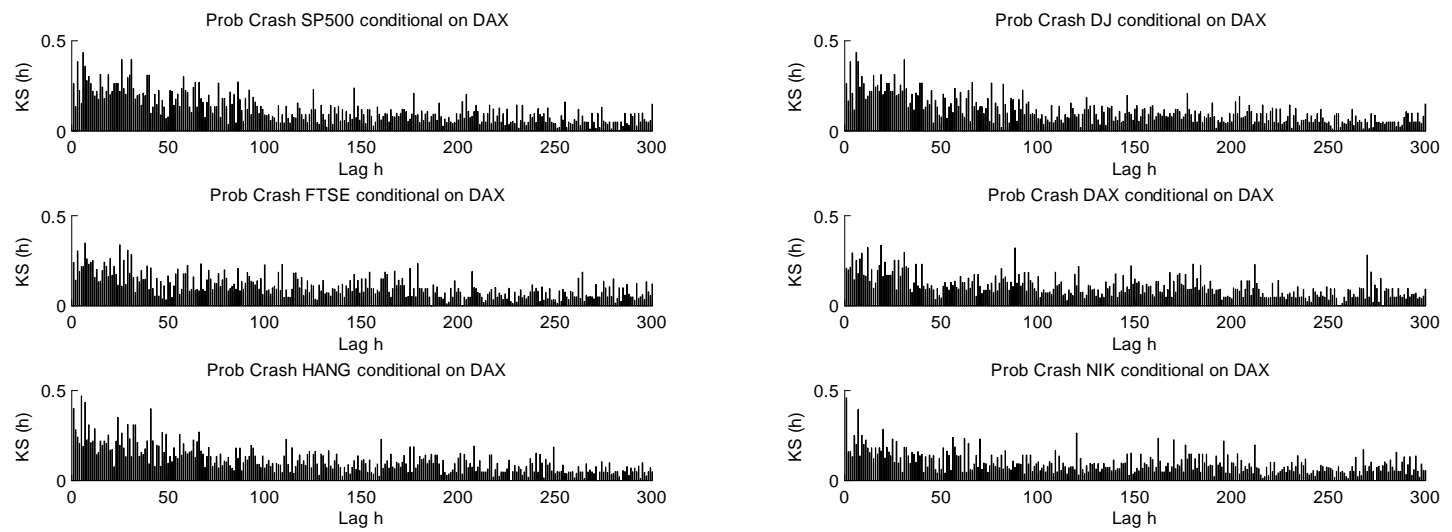

FiguRE 7: Estimated maximal conditional probabilities of crashes conditioning on DAX for quantiles $1 \%$ to $5 \%$. 

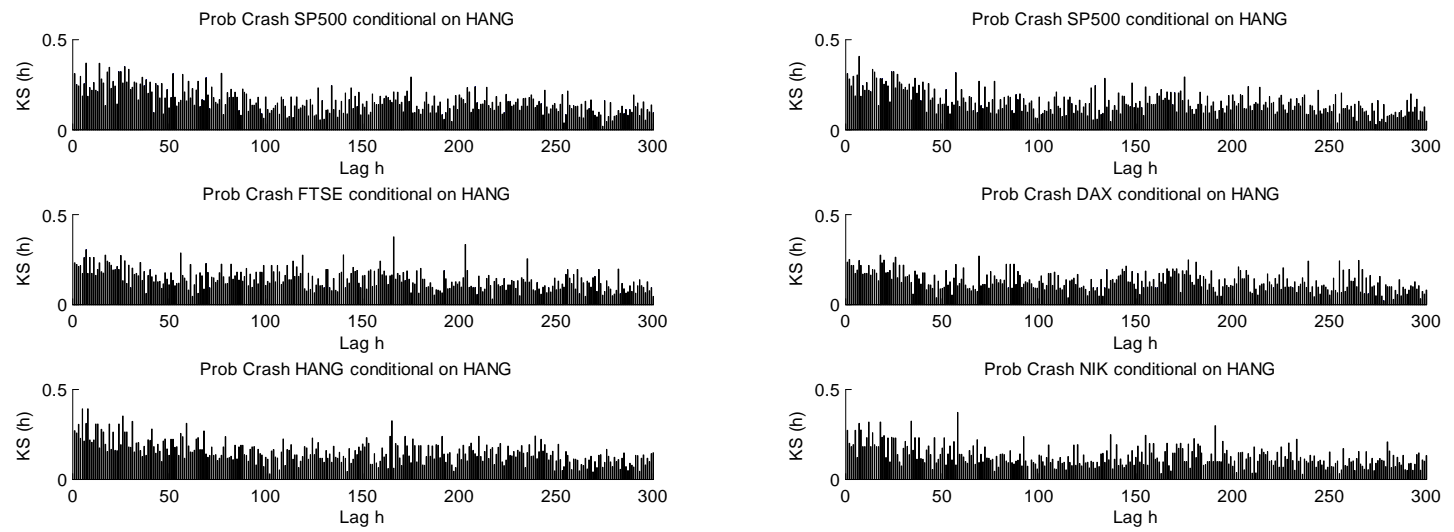

FIGURE 8: Estimated maximal conditional probabilities of crashes conditioning on HANG for quantiles $1 \%$ to $5 \%$.
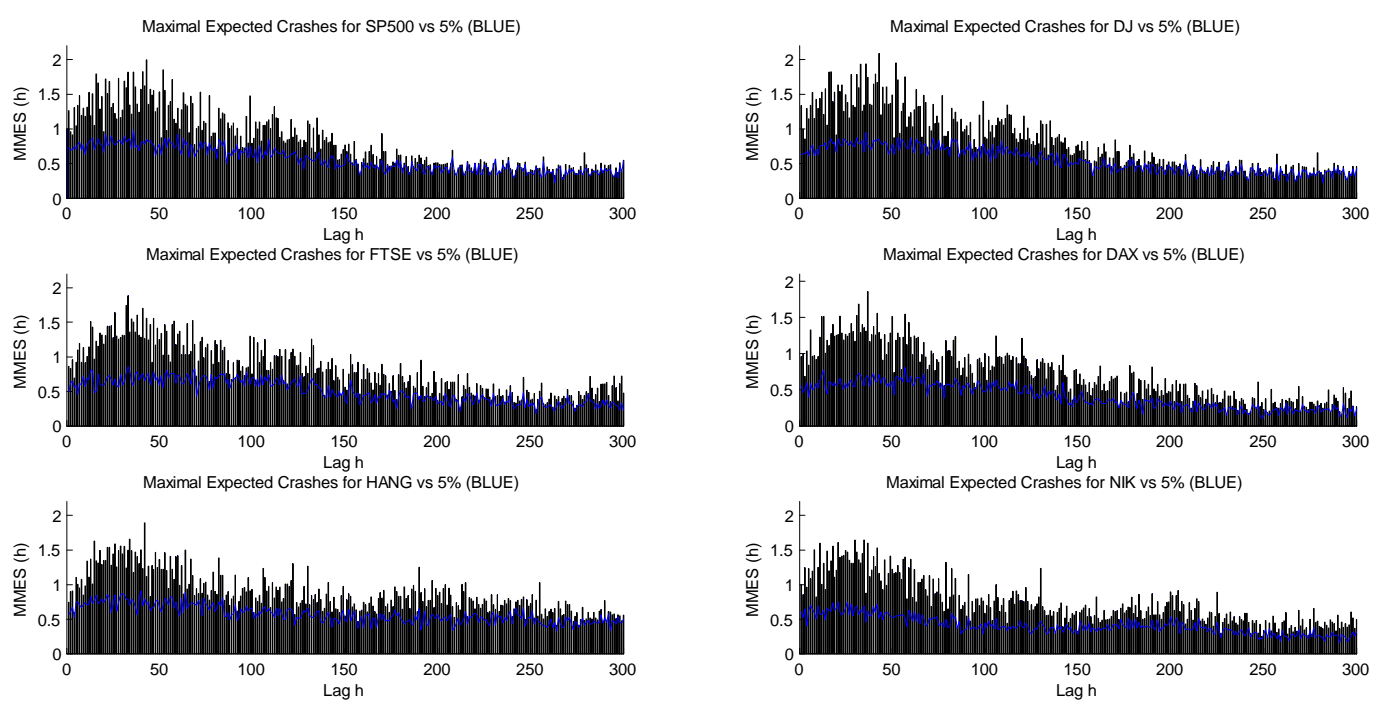

FiguRE 9: Estimated maximal number of crashes conditional on quantiles $1 \%$ to $5 \%$ for different stocks. Blue lines denote estimated number of crashes conditional on quantile $5 \%$ only. 